\title{
STRATIFICATIONS OF MARSDEN-WEINSTEIN REDUCTIONS FOR REPRESENTATIONS OF QUIVERS AND DEFORMATIONS OF SYMPLECTIC QUOTIENT SINGULARITIES
}

\author{
MAURIZIO MARTINO
}

\begin{abstract}
We investigate the Poisson geometry of the Marsden-Weinstein reductions of the moment map associated to the cotangent bundle of the space of representations of a quiver. We show that the stratification by representation type equals the stratification by symplectic leaves. The deformed symplectic quotient singularities - spectra of centres of symplectic reflection algebras associated to a wreath product - are shown to be isomorphic to reductions of a certain quiver. This establishes a method to calculate when these deformations are smooth. Furthermore, the isomorphism identifies symplectic leaves and so one can give a description of their symplectic leaves in terms of roots of the quiver.
\end{abstract}

\section{INTRODUCTION}

1.1. The representation theory of quivers is a fundamental topic in modern algebra. An interesting area in this subject has been the study of the geometry of the moment map, or, equivalently, the representation spaces of deformed preprojective algebras, with applications to Kleinian singularities and differential equations. Many interesting results have been obtained using the combinatorics of root systems for quivers, see [7] for example. We take a different point of view and examine the symplectic geometry of the moment map. In particular we describe the symplectic leaves of these Marsden-Weinstein reductions.

We apply these results to symplectic reflection algebras. These were introduced by Etingof and Ginzburg in [13] and are a beautiful class of algebras which have connections with algebraic geometry, integrable systems and combinatorics. At parameter $t=0$ the spectra of the centres of these algebras are Poisson deformations of symplectic quotient singularities. The symplectic leaves tell us valuable information about these varieties and also about the representation theory of the corresponding symplectic reflection algebras, 3]. By using quivers we can calculate, in a large class of examples, the symplectic leaves in terms of the relatively well understood theory of roots.

1.2. We summarise our results; full details and definitions are given in later chapters. Let $Q$ be a quiver with vertex set $I$ and set of arrows, $A$, and let $\bar{Q}$ be the double quiver of $Q$. Let $\lambda \in \mathbb{C}^{I}$. Then we define the deformed preprojective algebra

$$
\Pi_{\lambda}=\frac{\mathbb{C} \bar{Q}}{\left(\sum_{a \in A}\left[a, a^{*}\right]-\sum_{i \in I} \lambda_{i} e_{i}\right)} .
$$

The variety

$$
\operatorname{Rep}\left(\Pi_{\lambda}, \alpha\right) \subset \bigoplus_{a \in \bar{A}} \operatorname{Mat}\left(\alpha_{h(a)} \times \alpha_{t(a)}, \mathbb{C}\right)=\operatorname{Rep}(\bar{Q}, \alpha)
$$


classifies the representations of $\Pi_{\lambda}$ with dimension vector $\alpha \in \mathbb{N}^{I}$ where $\lambda \cdot \alpha=0$. The group

$$
\mathrm{G}(\alpha)=\left(\prod_{i \in I} \mathrm{GL}\left(\alpha_{i}, \mathbb{C}\right)\right) / \mathbb{C}^{\times}
$$

acts on $\operatorname{Rep}\left(\Pi_{\lambda}, \alpha\right)$ by basechange. The points of the algebraic quotient,

$$
\mathcal{N}(\lambda, \alpha):=\operatorname{Rep}\left(\Pi_{\lambda}, \alpha\right) / / \mathrm{G}(\alpha),
$$

correspond to isomorphism classes of semisimple representations of $\Pi_{\lambda}$ of dimension $\alpha$. If $M$ is a semisimple $\Pi_{\lambda}$-module then we can decompose it into its simple components $M=$ $M_{1}^{\oplus k_{1}} \oplus \cdots \oplus M_{r}^{\oplus k_{r}}$ where the $M_{t}$ are non-isomorphic simples. If $\beta^{(t)}$ is the dimension vector of $M_{t}$, then we say $M$ has representation type

$$
\left(k_{1}, \beta^{(1)} ; \ldots ; k_{r}, \beta^{(r)}\right) .
$$

One can therefore stratify $\mathcal{N}(\lambda, \alpha)$ by representation type.

1.3. The variety $\mathcal{N}(\lambda, \alpha)$ occurs as the quotient of a fibre of the moment map and therefore has the structure of a Poisson variety. Any Poisson variety decomposes into the disjoint union of symplectic leaves, see [3]. For $\mathcal{N}(\lambda, \alpha)$ we show that this has a straightforward description.

Theorem. The stratification of $\mathcal{N}(\lambda, \alpha)$ by representation type equals the stratification by symplectic leaves.

We note that one can describe $\mathcal{N}(\lambda, \alpha)$ as a version of the quiver varieties introduced by Nakajima, Remark 6.7. Our approach of considering the Poisson geometry of these reductions has been put into a more general framework through recent work of [9] and [30].

1.4. We apply Theorem [1.3 to symplectic reflection algebras, these are defined in Section 7. Let $\Gamma$ be a finite subgroup of $S L(2, \mathbb{C})$ and let $n>1$ be an integer. Let $H_{\mathbf{c}}$ be the symplectic reflection algebra (at parameter $t=0$ ) for the wreath product $\Gamma_{n}=S_{n} \ltimes \Gamma^{n}$, and let $Z_{\mathbf{c}}$ denote its centre. The variety $\operatorname{Max} Z_{\mathbf{c}}$ is Poisson. The connection to quivers is via the McKay correspondence: to the group $\Gamma$ we associate a quiver, $Q_{\Gamma}$, by choosing an orientation of its McKay graph. The quiver, $Q$, is obtained from $Q_{\Gamma}$ by adding one vertex and one arrow from this vertex to an extending vertex of $Q_{\Gamma}$. We establish the following.

Theorem. For the quiver $Q$, a dimension vector $\alpha$ and a parameter $\lambda \in \mathbb{C}^{I}$ depending on $\mathbf{c}$

$$
\mathcal{N}(\lambda, \alpha) \cong \operatorname{Max} Z_{\mathbf{c}}
$$

This isomorphism is Poisson up to nonzero scalar multiple and in particular maps symplectic leaves to symplectic leaves.

Crawley-Boevey and Holland proved this theorem for finite subgroups of $\mathrm{SL}(2, \mathbb{C})$, see [10], Theorem 0.2], although they do not mention Poisson structures, and our result can be seen as an extension of theirs. In fact, our proof is based on a result of Etingof and Ginzburg [13. Theorem 11.16] who prove Theorem 7.4 with the extra condition that the parameter c is generic. The definition of the dimension vector $\alpha$ and deformation parameter $\lambda$ used in the above theorem are given in 6.7 where they are written $\epsilon_{\infty}+n \delta$ and $\lambda^{\prime}(\mathbf{c})$ respectively. 
1.5. The proof of Theorem 1.4 is rather complicated and involves several stages. The Calogero-Moser space, $X_{\mathbf{c}} / / \mathrm{G}(n \delta)$, defined in 6.2. plays an important role as does its equivalent description $\hat{X}_{\mathbf{c}} / / \hat{\mathrm{G}}(n \delta)$, see 6.5. We can break down the isomorphism of Theorem 1.4 into the sequence of isomorphisms. In the diagram below the superscripts indicate the subsection in which the corresponding isomorphism is proved:

$$
\mathcal{N}(\lambda, \alpha) \stackrel{6.10}{\sim} \hat{X}_{\mathbf{c}} / / \hat{\mathrm{G}}(n \delta) \stackrel{6.6}{\sim} X_{\mathbf{c}} / / \mathrm{G}(n \delta) \stackrel{\text { 7.4 }}{\sim} \operatorname{Max} Z_{\mathbf{c}}
$$

All of the above maps are Poisson except the final one which is only Poisson up to nonzero scalar multiple.

1.6. The symplectic leaves of $\operatorname{Max} Z_{\mathbf{c}}$ are an important in the representation theory of $H_{\mathbf{c}}$. Brown and Gordon proved in [3. Theorem 4.2] that if two maximal ideals $\mathfrak{m}$ and $\mathfrak{n}$ of $Z_{\mathbf{c}}$ lie in the same symplectic leaf then the factor algebras $H_{\mathbf{c}} / \mathfrak{m} H_{\mathbf{c}}$ and $H_{\mathbf{c}} / \mathfrak{n} H_{\mathbf{c}}$ are isomorphic. Therefore understanding the leaves is useful in studying the irreducible representations of $H_{\mathbf{c}}$. We have the following consequence of Theorems 1.3 and 1.4

Theorem. There is a one-to-one correspondence between the symplectic leaves of $\operatorname{Max} Z_{\mathbf{c}}$ and the representation types of $\mathcal{N}(\lambda, \alpha)$.

Therefore, using Crawley-Boevey's work on root systems and dimension vectors of simple modules for $\Pi_{\lambda}$, [7, one can use linear algebra to calculate information about the symplectic leaves of $\operatorname{Max} Z_{\mathbf{c}}$, see 5.12

1.7. We organise our paper as follows. In Section 2 we recall facts about Poisson varieties and symplectic leaves. The moment map and the Marsden-Weinstein reduction procedure is discussed in Section 3. We examine the special case of reductions for symplectic vector spaces with a hyper-Kähler structure in Section 4 , identifying the symplectic leaves in Proposition 4.9. We then apply these to results to representations of deformed preprojective algebras in Section 5 to prove Theorem 1.3. The proof of Theorem 1.4 occupies Sections 6 and 7 in which we also introduce Calogero-Moser space. We calculate the example of the centre of the rational Cherednik algebra of type $B_{n}$ in Section 8, computing the parameters at which $\operatorname{Max} Z_{\mathbf{c}}$ is singular and also the number of symplectic leaves and their dimensions.

\section{Poisson VARIETIES AND SyMPleCtiC LEAVES}

2.1. Poisson algebras. Throughout we work over the complex numbers. Thus varieties and manifolds are complex, and symplectic form will mean complex symplectic form, unless stated otherwise. In this section we introduce the notions of Poisson varieties and manifolds and the stratification by symplectic leaves.

Let $A$ be a commutative $\mathbb{C}$-algebra. $A$ is a Poisson algebra if there exists a $\mathbb{C}$-bilinear bracket $\{-,-\}: A \times A \rightarrow A$ such that

(1) $(A,\{-,-\})$ is a Lie algebra;

(2) $\{-,-\}$ satisfies the product rule, that is, $\{a, b c\}=\{a, b\} c+b\{a, c\}$ for all $a, b, c \in A$.

Let $I$ be an ideal of a Poisson algebra $R$. Then $I$ is a Poisson ideal if $\{R, I\} \subseteq I$. A homomorphism $\phi: A \rightarrow B$ between Poisson algebras $\left(A,\{-,-\}_{A}\right)$ and $\left(B,\{-,-\}_{B}\right)$ is called Poisson if $\phi\left\{a_{1}, a_{2}\right\}_{A}=\left\{\phi\left(a_{1}\right), \phi\left(a_{2}\right)\right\}_{B}$ for all $a_{1}, a_{2} \in A$. 
2.2. Poisson varieties. We say that an algebraic variety, $X$, with structure sheaf $\mathcal{O}$, is a Poisson variety if $\mathcal{O}$ is a sheaf of Poisson algebras such that the restriction homomorphisms are Poisson. As an example, given any finitely generated Poisson algebra $A$, let $\operatorname{Max} A$ denote its set of maximal ideals endowed with the structure of an algebraic variety. Then $\operatorname{Max} A$ is a Poisson variety, as is any open subvariety of Max $A$. We say that a morphism $\psi: X \rightarrow Y$ between Poisson varieties $X$ and $Y$ is Poisson if for all open $U \subseteq Y, \psi_{U}^{\sharp}$ : $\mathcal{O}_{Y}(U) \rightarrow \mathcal{O}_{X}\left(\psi^{-1}(U)\right)$ is Poisson. If $X$ and $Y$ are both affine varieties then $\psi$ is Poisson if and only if the comorphism $\psi^{\sharp}: \mathcal{O}_{Y}(Y) \rightarrow \mathcal{O}_{X}(X)$ is a Poisson homomorphism. For any $f \in \mathcal{O}(X)$ the Hamiltonian vector field of $f$ is the derivation, $\{f,-\}$, of $\mathcal{O}(X)$.

2.3. Let $M$ be a smooth affine algebraic variety, and denote its structure sheaf by $\mathcal{O}$. We say that $M$ is a symplectic variety if it comes equipped with nondegenerate closed 2form, $\omega$. A symplectic variety $(M, \omega)$ gives rise to a Poisson bracket on $\mathcal{O}(M)$ as follows. Let $\operatorname{Vect}(M)$ denote the Lie algebra of vector fields on $M$. Nondegeneracy of $\omega$ means that there is a $\mathbb{C}$-linear map $\mathcal{O}(M) \rightarrow \operatorname{Vect}(M) ; f \mapsto \Xi_{f}$ where $\Xi_{f}$ is the unique vector field such that $\omega\left(\Xi_{f},-\right)=d f$. For all $f, g \in \mathcal{O}(M)$ we define the bracket of $f$ and $g$ by $\{f, g\}=\Xi_{f} g=\omega\left(\Xi_{f}, \Xi_{g}\right)$. This is a Poisson bracket by [21, Proposition 5.5.3]. Hence $M$ is a Poisson variety and $\Xi_{f}$ is the Hamiltonian vector field of $f$, see 2.1 above.

2.4. Poisson manifolds. For a manifold, $M$, we denote the algebra of holomorphic functions defined on $M$ by $\mathcal{C}(M)$. We say that $M$ is Poisson manifold if $\mathcal{C}(M)$ is a Poisson algebra. A smooth map $f: M \rightarrow N$ between Poisson manifolds is called Poisson if the corresponding algebra homomorphism $f^{*}: \mathcal{C}(N) \rightarrow \mathcal{C}(M)$ is a Poisson homomorphism. Any symplectic manifold is a Poisson manifold - the argument for showing that symplectic varieties are Poisson varieties works for manifolds also. For any $f \in \mathcal{C}(M)$ we define the Hamiltonian vector field of $f$ to be the derivation of $\mathcal{C}(M)$ given by $\{f,-\}$.

A smooth Poisson variety, $X$, is naturally a manifold. The existence of a Poisson bracket on $\mathcal{O}(X)$ is equivalent to the existence of a bivector $\theta: X \rightarrow T^{2} X$ which is a section to the bundle map and satisfies $[\theta, \theta]=0$. Here $T X$ is the tangent bundle of $X$ and $[-,-]$ is the Schouten bracket. Considering the bivector as complex analytic yields a Poisson bracket on $\mathcal{C}(X)$, making $X$ a Poisson manifold.

\subsection{Let $M$ be a Poisson manifold.}

Definition. The symplectic leaf $\mathcal{S}(p)$ containing a point $p$ of $M$ is the set of points $q$ which are connected to $p$ by piecewise smooth curves, each segment of which is the integral curve of a Hamiltonian vector field.

Therefore to find the symplectic leaf containing $p$ one works out all the points one can reach by travelling along integral curves of Hamiltonian vector fields at $p$. For each such point, $q$, connected to $p$ in this way one repeats the process, finding the points one can reach by travelling along integral curves of Hamiltonian vector fields at $q$. Then one continues this process until the symplectic leaf is swept out.

2.6. The rank of $p$ is equal to the dimension of the subspace of $T_{p} M$ spanned by the Hamiltonian vector fields evaluated at $p$. For Poisson manifolds $M$ and $N$ such that $N \subseteq M$ we shall say that $N$ is a Poisson submanifold of $M$ if the inclusion map is Poisson.

The proof of the following theorem, under some additional hypotheses, goes back to Lie, and the general case is due to Kirillov, [17] for a proof in the complex case see 1, Theorem 
3.26]. Further discussion about symplectic leaves can be found in [21, Section 10] and in 31 .

Theorem. $M$ is a disjoint union of its symplectic leaves. Each leaf is a symplectic manifold which is a Poisson submanifold of $X$ and the dimension of the leaf through $p$ is equal to the rank of $p$ in $M$.

2.7. Suppose that $X$ is a smooth affine Poisson variety. Then, as described in 2.4. $X$ is a Poisson manifold so one can stratify $X$ by symplectic leaves. Although $X$ is an algebraic variety it is quite possible that the leaves are not algebraic varieties, see [3, Remarks 3.6 (1)].

Now suppose that $X$ is not necessarily smooth; we can stratify $X$ by symplectic leaves, as described in [3. §3.5], as follows. Let $U_{0}$ be the smooth locus of $X$. Then, since $X$ is affine, $U_{0}$ is a smooth Poisson variety and so is a Poisson manifold. We can stratify $U_{0}$ by symplectic leaves, say $U_{0}=\bigsqcup_{i \in \mathcal{I}_{0}} \mathcal{S}_{i, 0}$. Now we proceed inductively by setting $X_{0}=X$ and defining $X_{k}=X_{k-1} \backslash U_{k-1}$ for $k \geq 1 . X_{k}$ is an affine Poisson variety by [23, Proposition 15.2.14(i)], so one can, as above, stratify the smooth locus, $U_{k}$, of $X_{k}$ by symplectic leaves, $U_{k}=\bigsqcup_{i \in \mathcal{I}_{k}} \mathcal{S}_{i, k}$. Then

$$
X=U_{0} \sqcup \cdots \sqcup U_{t}
$$

for some $t$ and we call

$$
X=\bigsqcup_{\substack{i \in \mathcal{I}_{k}, 0 \leq k \leq t}} \mathcal{S}_{i, k}
$$

the stratification of $X$ by symplectic leaves.

2.8. We say that a closed subvariety, $Y$, of $X$ is Poisson if the inclusion $Y \hookrightarrow X$ is Poisson. This is equivalent to the condition that the defining ideal of $Y$ is a Poisson ideal.

Proposition. 3, Proposition 3.7] If $Y$ is a closed Poisson subvariety of $X$ and $\mathcal{S}$ is a symplectic leaf of $X$ then $\mathcal{S} \cap Y \neq \emptyset$ implies that $\mathcal{S} \subseteq Y$. Furthermore, if $X$ is a finite union of symplectic leaves then each leaf is an irreducible locally closed smooth subvariety of $X$.

\section{THE MOMENT MAP}

3.1. For details of the following see [6, $\S 1.4]$. Let $(M, \omega)$ be a symplectic variety as in 2.3 and suppose that a reductive algebraic group $G$ acts by morphisms on $M$ preserving the symplectic form. Let $\mathfrak{g}$ be the Lie algebra of $G$.

A vector field $X$ is symplectic if it preserves the symplectic form on $M$, that is, $L_{X} \omega=0$, where $L_{X}$ is the Lie derivative with respect to $X$. We denote by $\operatorname{Symp}(M)$ the Lie subalgebra of symplectic vector fields on $M$.

Proposition. 6. Proposition 1.2.5] $\Xi_{f}$ is a symplectic vector field for all $f \in \mathcal{O}(M)$, and the map $f \mapsto \Xi_{f}$ defines a Lie algebra homomorphism $(\mathcal{O}(M),\{-,-\}) \rightarrow(\operatorname{Symp}(M),[-,-])$.

Recall the infinitesimal action of $\mathfrak{g}$ on $\mathcal{O}(M)$ : for each $x \in \mathfrak{g}$ and $f \in \mathcal{O}(M), x_{M}(f):=$ $\left.\frac{d}{d t}(\exp (t x) \circ f)\right|_{t=0}$. The operator $x_{M}$ is a derivation of $\mathcal{O}(M)$ and so defines a map $\mathfrak{g} \rightarrow$ Vect $(M)$. This map is a Lie algebra homomorphism, [21, Proposition 9.3.6], and one can easily check that its image is contained in the space of symplectic vector fields.

The $G$-action is said to be Hamiltonian if there exists a Lie algebra homomorphism $H$ : $\mathfrak{g} \rightarrow(\mathcal{O}(M),\{-,-\})$ such that the following diagram commutes: 


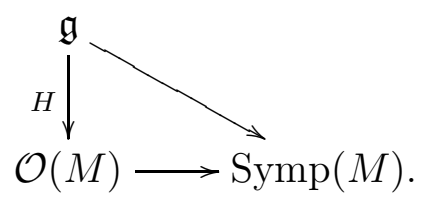

Suppose that the $G$-action is Hamiltonian and let $H_{x}=H(x)$ for all $x \in \mathfrak{g}$. The moment map $\mu: M \rightarrow \mathfrak{g}^{*}$ is the morphism of algebraic varieties defined by $\mu(m)(x)=H_{x}(m)$ for all $m \in M$ and $x \in \mathfrak{g}$.

Example. Recall that the coordinate ring, $\mathcal{O}\left(\mathfrak{g}^{*}\right)$, of $\mathfrak{g}^{*}$, equals the symmetric algebra $S \mathfrak{g}$ of $\mathfrak{g}$. The algebra $S \mathfrak{g}$ has a canonical Poisson bracket induced from the Lie bracket on $\mathfrak{g}$. One can calculate the infinitesimal action of $\mathfrak{g}$ on $S \mathfrak{g}$ (see [21, Examples (c), Page 272): for $x \in \mathfrak{g}$ and $f \in \mathfrak{g}, x_{\mathfrak{g}^{*}}(f)=[x, f]$. The action of $G$ is Hamiltonian, with $H_{x}=x$ for all $x \in \mathfrak{g}$, and the corresponding moment map is $\mu=\operatorname{Id}_{\mathfrak{g}^{*}}$.

Let $\mathcal{L}$ be a closed orbit (under the coadjoint action) in $\mathfrak{g}^{*}$. Let $I$ be the defining ideal of $\mathcal{L}$. Then $I$ is a Poisson ideal of $S \mathfrak{g}$ so that $\mathcal{L}$ is a Poisson variety. In fact this makes $\mathcal{L}$ a symplectic variety, [6, Proposition 1.3.21]. Now for all $x \in \mathfrak{g}, x_{\mathcal{L}}$ is the derivation of $\mathcal{O}(\mathcal{L})=S \mathfrak{g} / I$ given by $x_{\mathcal{L}}(f+I)=[x, f]+I$ for all $f \in \mathfrak{g} \subset S \mathfrak{g}$. One sees that the action of $G$ on $\mathcal{L}$ is Hamiltonian with $H_{x}=x+I$ and the corresponding moment map is simply the inclusion $\mathcal{L} \hookrightarrow \mathfrak{g}^{*}$.

3.2. The moment map has the following properties.

Proposition. [6, Lemma 1.4.2] The map

$$
\mu^{\sharp}: \mathbb{C}[\mathfrak{g}] \rightarrow \mathcal{O}(M)
$$

induced by $\mu$ is a Poisson homomorphism, and if $G$ is connected then $\mu$ is G-equivariant (relative to the coadjoint action on $\mathfrak{g}^{*}$ ).

3.3. In general it is interesting to know whether a group action on a symplectic variety is Hamiltonian. In the linear case this is known.

Theorem. [6, Proposition 1.4.6] Let $V$ be a symplectic vector space with symplectic form $\omega$ and let $G$ be a reductive algebraic subgroup of $\mathrm{Sp} V$. Then the action is Hamiltonian with $H_{A}(v)=\frac{1}{2} \omega(A \cdot v, v)$ for all $A \in$ Lie $G$ and $v \in V$ and the corresponding moment map is G-equivariant.

3.4. Reduction. Many of the varieties which appear in this paper arise as quotient spaces of fibres of a moment map, and we shall see now how these spaces carry a Poisson structure. Let $(M, \omega)$ be a symplectic variety and let the reductive algebraic group $G$ act by morphisms on $M$ preserving $\omega$. Suppose this action is Hamiltonian and that the corresponding moment map $\mu$ is $G$-equivariant. Let $\mathcal{L}$ be a closed orbit under the coadjoint action of $G$ on $\mathfrak{g}^{*}$, with defining $G$-stable ideal $J \triangleleft \mathbb{C}[\mathfrak{g}]$. Then $\mu^{-1}(\mathcal{L})$ is a $G$-stable closed subvariety of $M$, with defining $G$-stable radical ideal $I \triangleleft \mathcal{O}(M)$.

We consider the quotient variety $M_{\mathcal{L}}:=\mu^{-1}(\mathcal{L}) / / G$, which we call a Marsden-Weinstein reduction. The double lines indicate that we consider closed orbits, in other words points in the maximal ideal spectrum of $\mathcal{O}\left(\mu^{-1}(\mathcal{L})\right)^{G}$. Let $\{-,-\}$ be the bracket on $\mathcal{O}(M)$. Since $G$ is reductive $(\mathcal{O}(M) / I)^{G} \cong \mathcal{O}(M)^{G} / I^{G}$ so one can define a bracket on $(\mathcal{O}(M) / I)^{G}$ by defining 
one on $\mathcal{O}(M)^{G} / I^{G}$. Define a bracket, $\{-,-\}^{\prime}$, on $\mathcal{O}(M)^{G} / I^{G}$ by $\left\{f+I^{G}, g+I^{G}\right\}^{\prime}=\{f, g\}+I^{G}$ for all $f, g \in \mathcal{O}(M)^{G}$.

Proposition. The bracket $\{-,-\}^{\prime}$ is well-defined and is a Poisson bracket on $\mathcal{O}\left(M_{\mathcal{L}}\right)$.

Proof. The bracket $\{-,-\}^{\prime}$ will clearly yield a Poisson bracket as long as it is well defined. To see this we note first that $\{f, g\} \in \mathcal{O}(M)^{G}$ for all $f, g \in \mathcal{O}(M)^{G}$. It remains to show that $I^{G}$ is a Poisson ideal of $\mathcal{O}(M)^{G}$.

For all $x \in \mathfrak{g}, \mu^{\sharp}(x)=x \circ \mu$ and if we evaluate this at an element of $m \in M$ we see that $(x \circ \mu)(m)=x\left(H_{-}(m)\right)=H_{x}(m)$. In short, $\mu^{\sharp}(x)=H_{x}$ and it follows that $I^{\prime}:=\mathcal{O}(M) \mu^{\sharp}(J)$ is generated by polynomials in the $H_{x}$.

Now, if $f \in \mathcal{O}(M)^{G}$ then $h \cdot f=f$ for all $h \in G$ and therefore $x_{M} f=0$ for all $x \in \mathfrak{g}$. Thus $\left\{H_{x}, f\right\}=x_{M} f=0$ and, by the product rule, $\{f, i\} \in I^{\prime}$ for all $i \in I^{\prime}$. Therefore $I^{\prime G}$ is a Poisson ideal of $\mathcal{O}(M)^{G}$. Finally, $I=\sqrt{I^{\prime}}$ implies that $I^{G}=\sqrt{I^{\prime G}}$, and so $I^{G}$ is a Poisson ideal of $\mathcal{O}(M)^{G}$ by [11, 3.3.2].

Example (Rank one matrices). Let $M_{n}=\operatorname{Mat}(n \times n, \mathbb{C})$ which is the Lie algebra of $\operatorname{GL}(n, \mathbb{C}) . \quad M_{n}$ can be identified with with its dual via the trace pairing and in this way becomes a Poisson variety as in Example 3.1. One can describe $\mathcal{O}\left(M_{n}\right)$ as $\mathbb{C}\left[x_{i j}: 1 \leq i, j \leq n\right]$ and then its Poisson bracket is $\left\{x_{i j}, x_{k l}\right\}=-\delta_{j k} x_{i l}+\delta_{i l} x_{k j}$.

Let $R_{l}$ be the subvariety of $M_{n}$ consisting of matrices whose rank is less than or equal to $l$. Thus $R_{l}$ is the set of matrices whose $(l+1) \times(l+1)$ minors are zero and so is a closed subvariety. The defining ideal of $R_{l}$ is the prime ideal, $I_{l}$, of $\mathbb{C}\left[x_{i j}\right]$ generated by the generic $(l+1) \times(l+1)$ minors, [4, Theorem 2.10]. We are interested in looking at the matrices whose rank is less than or equal to one, that is, the subvariety $R_{1}$. By [23, Proposition 15.2.14], $I_{1}$ is a Poisson ideal.

Let $0 \neq k \in \mathbb{C}$ and let $T_{k} \subset M_{n}$ be the closed subvariety of matrices whose trace equals $k$. Then $T_{k}$ has defining ideal $\left(x_{11}+\cdots+x_{n n}-k\right) \triangleleft \mathbb{C}\left[x_{i j}\right]$ and a direct calculation shows that this a Poisson ideal. From this it follows that $U_{k}:=R_{1} \cap T_{k}$ is a closed Poisson subvariety of $M_{n}$ with defining ideal $J_{k}:=\sqrt{I_{1}+\left(x_{11}+\cdots+x_{n n}-k\right)}$.

We can describe $U_{k}$ as a reduction for a moment map, as follows. Let $V$ be an $n$ dimensional complex vector space and consider the symplectic space $W=V \oplus V^{*}$. Using the usual scalar product on $V$ we can think of $V$ as consisting of column vectors and $V^{*}$ of row vectors. We fix bases $c_{1}, \ldots, c_{n} \in V$ and $r_{1}, \ldots, r_{n} \in V^{*}$. There is an action of $\mathbb{C}^{\times}$by $\lambda \cdot(c, r)=\left(\lambda v, \lambda^{-1} r\right)$ for all $\lambda \in \mathbb{C}^{\times}, c \in V$ and $r \in V^{*}$. This action is Hamiltonian, Theorem 3.3. with moment map $\mu: W \rightarrow \mathbb{C} ; \mu(c, r)=r c$, here we identify $\mathbb{C}$ with $\mathbb{C}^{*}=\operatorname{Lie}\left(\mathbb{C}^{\times}\right)^{*}$ via the trace pairing. It is an easy calculation to see that

$$
\mathcal{O}(W)^{\mathbb{C}^{\times}}=\left(\mathcal{O}(V) \otimes \mathcal{O}\left(V^{*}\right)\right)^{\mathbb{C}^{\times}}=\mathbb{C}\left[r_{i} \otimes c_{j}: 1 \leq i, j \leq n\right]
$$

and then it follows that

$$
\mathcal{O}\left(\mu^{-1}(k) / / \mathbb{C}^{\times}\right)=\mathbb{C}\left[r_{i} \otimes c_{j}\right] / I_{k}
$$

where $I_{k}:=\sqrt{\left(r_{1} \otimes c_{1}+\cdots+r_{n} \otimes c_{n}-k\right)} \triangleleft \mathbb{C}\left[r_{i} \otimes c_{j}\right]$. One can check directly that $I_{k}$ is a Poisson ideal of $\mathbb{C}\left[r_{i} \otimes c_{j}\right]$, which verifies Proposition 3.4 in this example.

We now describe the isomorphism between $\mu^{-1}(k) / / \mathbb{C}^{\times}$and $U_{k}$. Let $G=\operatorname{GL}(n, \mathbb{C})$ which acts naturally on $V$ and by conjugation on $M_{n} \cong \operatorname{Lie}(G)^{*}$. One obtains an action of $G$ on $W$ and the corresponding moment map (Theorem 3.3) is

$$
\nu: W \rightarrow M_{n} ;(r, c) \mapsto r \otimes c \in V \otimes V^{*} \cong M_{n} .
$$


It is clear that the image of $\nu$ is contained in $R_{1}$. The map $\nu$ is equivariant for the action of $G$ and therefore also for the action of $\mathbb{C}^{\times}$, where $\mathbb{C}^{\times}$is thought of as the subgroup of nonzero scalar matrices in $G$. Therefore, since $\mathbb{C}^{\times}$acts trivially on $M_{n}$ we get a morphism induced from $\nu$ :

$$
t: \mu^{-1}(k) / / \mathbb{C}^{\times} \rightarrow U_{k}
$$

whose comorphism is the map

$$
\mathbb{C}\left[x_{i j}\right] / J_{k} \rightarrow \mathbb{C}\left[r_{i} \otimes c_{j}\right] / I_{k} ; x_{i j}+J_{k} \mapsto r_{i} \otimes c_{j}+I_{k}
$$

This is a Poisson isomorphism and so we have described $U_{k}$ as the reduction $\mu^{-1}(k) / / \mathbb{C}^{\times}$.

3.5. Given a reduction over a closed coadjoint orbit, one can perform the so-called shifting trick to express this as reduction over a fixed point.

Lemma (The shifting trick). Let $\mathcal{L}$ be a closed coadjoint orbit of $\mathfrak{g}^{*}$ and let $\xi \in \mathfrak{g}^{*}$ be a fixed point. Then $M \times-\mathcal{L}$ is a symplectic variety such that the natural action of $G$ is Hamiltonian with corresponding moment map $\mu^{\prime}: M \times-\mathcal{L} \rightarrow \mathfrak{g}^{*} ;(m, x) \mapsto \mu(m)+x$. There is an isomorphism of Poisson varieties $\mu^{\prime-1}(\xi) / / G \cong \mu^{-1}(\mathcal{L}+\xi) / / G$.

Proof. As explained in Example $3.1-\mathcal{L}$ is a symplectic variety with Hamiltonian $G$-action such that the moment map is just the inclusion $-\mathcal{L} \hookrightarrow \mathfrak{g}^{*}$. Therefore the product $M \times-\mathcal{L}$ is symplectic and the action of $G$ on this product is Hamiltonian because it is Hamiltonian on each component. To see this one takes the Hamiltonian maps $H_{1}, H_{2}$ of $M$ and $-\mathcal{L}$ respectively and checks that the map $H=H_{1} \otimes 1+1 \otimes H_{2}: \mathfrak{g} \rightarrow \mathcal{O}(M \times-\mathcal{L})=\mathcal{O}(M) \otimes \mathcal{O}(-\mathcal{L})$ is a Hamiltonian for the action of $G$ on the product. The moment map corresponding to $H$ is then the sum of the moment maps for $M$ and $-\mathcal{L}$. The projection map $M \times-\mathcal{L} \rightarrow M$ is a $G$ equivariant Poisson map and restricts to an isomorphism between $\mu^{\prime-1}(\xi)$ and $\mu^{-1}(\mathcal{L}+\xi)$. The $G$-equivariance of the projection map means that this induces an isomorphism $\mu^{\prime-1}(\xi) / / G \cong$ $\mu^{-1}(\mathcal{L}+\xi) / / G$. This isomorphism is Poisson because the projection map is Poisson and by the proposition above.

\section{THE LOCAL NORMAL FORM FOR THE MOMENT MAP}

4.1. We discuss a very particular case of reduction which will apply to representations of preprojective algebras. Our arguments follow closely those of [29] and [26, Section 3]. Let $M$ be a finite dimensional complex vector space. Let $G$ be a complex connected reductive algebraic group acting linearly on $M$, and we think of $G$ as the complexification of a compact real algebraic group $R$. Let $I: M \rightarrow M$ be the map defined by multiplication by $\sqrt{-1}$ and let $(-,-)$ be a real inner product on $M$ which is invariant under the actions of $I$ and $R$. Thus $M$ is a Kähler manifold. Let $J, K: M \rightarrow M$ be $\mathbb{R}$-linear maps preserving $(-,-)$ and satisfying $I^{2}=J^{2}=K^{2}=I J K=-\mathrm{Id}$. This makes $M$ into a hyper-Kähler manifold, [16].

4.2. We have real symplectic forms on $M$ given by $\omega_{1}(v, w)=(v, I w), \omega_{2}(v, w)=(v, J w)$ and $\omega_{3}(v, w)=(v, K w)$ for all $v, w \in M$. If we define $\omega=\omega_{2}+I \omega_{3}$ then this is a complex symplectic form on $M$. We shall assume that the action of $G$ preserves $\omega$. Let $\mathfrak{r}, \mathfrak{g}$ be the Lie algebras of $R$ and $G$ respectively. We have two moment maps to consider in this situation. The first, $\mu_{1}: M \rightarrow \mathfrak{r}^{*}$, is a moment map with respect to $\omega_{1}$ and is obtained completely analogously to that of Theorem 3.3 . We also have the moment map $\mu: M \rightarrow \mathfrak{g}^{*}$ with respect to $\omega$ which is constructed as in Theorem 3.3 . 
4.3. We wish to consider a closed orbit corresponding to a point in $\mu^{-1}(0) / / G$, say $G m$, for some $m \in \mu^{-1}(0)$. Let $H$ be the stabiliser of $m$ in $G$, and denote its Lie algebra by $\mathfrak{h}$. Let $T_{m}(G m)$ be the tangent space of $m$ in $G m$, and let $\left(T_{m}(G m)\right)^{\omega}=\left\{v \in T_{m} M\right.$ : $\omega(v, w)=0$ for all $\left.w \in T_{m}(G m)\right\}$. By [15, page 324] $T_{m}(G m) \subseteq\left(T_{m}(G m)\right)^{\omega}$; set $\hat{M}=$ $\left(T_{m}(G m)\right)^{\omega} / T_{m}(G m)$. The action of $H$ preserves the induced symplectic structure on $\hat{M}$, let $\hat{\mu}: \hat{M} \rightarrow \mathfrak{h}^{*}$ be the corresponding $H$-equivariant moment map, Theorem 3.3

We choose an $\operatorname{Ad}(H)$-invariant splitting $\mathfrak{g}=\mathfrak{h} \oplus \mathfrak{h}^{\perp}$ and a dual splitting $\mathfrak{g}^{*}=\mathfrak{h}^{*} \oplus \mathfrak{h}^{\perp *}$. There is a natural action of the reductive group, $H$, on $\mathfrak{h}^{\perp *} \times \hat{M}$ by $h \cdot(\xi, \hat{m})=\left(\operatorname{Ad}^{*}(h) \xi, h \hat{m}\right)$. The associated bundle $\left(G \times \mathfrak{h}^{\perp *} \times \hat{M}\right) / / H$ is a symplectic variety with Hamiltonian $G$-action (see [26, Page 164]) - the $G$-action is given by $x \cdot[g, \xi, \hat{m}]=[x g, \xi, \hat{m}]$ for all $x \in G$. The ( $G$-invariant) moment map is given by

$$
J(H \cdot(g, \xi, \hat{m}))=\operatorname{Ad}^{*}(g)(\xi+\hat{\mu}(\hat{m})) .
$$

4.4. We shall refer to the following theorem by saying that there exists a local normal form for $\mu$. Let $\Upsilon=\left(G \times \mathfrak{h}^{\perp *} \times \hat{M}\right) / / H$.

Theorem. [26. Lemma 3.2.1] Let $\pi$ and $\rho$ be the G-orbit maps for $M$ and $\Upsilon$ respectively. Let $y=\pi(m)$. Then there exist complex neighbourhoods, $U$, of $y$ and $W$ in $\left(\mathfrak{h}^{\perp *} \times \hat{M}\right) / / H$ and biholomorphisms $\Psi, \tilde{\Psi}$ such that the following diagram commutes

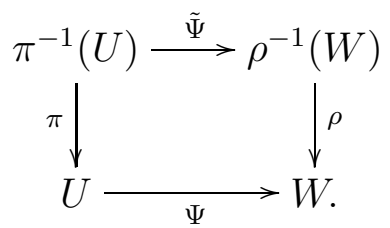

Furthermore, $\tilde{\Psi}$ is a G-equivariant map which intertwines symplectic forms and maps $m$ to $(H, 0,0) \in \Upsilon$.

4.5. Stratifying by orbit type. Let $\mathcal{L}$ be a closed coadjoint orbit in $\mathfrak{g}^{*}$ and let $Z=\mu^{-1}(\mathcal{L})$. Let $M_{\mathcal{L}}$ denote the corresponding Marsden-Weinstein reduction, $Z / / G$. Let $\pi: Z \rightarrow M_{\mathcal{L}}$ denote the orbit map.

One can stratify $M_{\mathcal{L}}$ in a natural way. For $z \in Z$ let $G_{z}$ be the stabiliser of $z$ in $G$. Let $\mathcal{T}$ be the set of conjugacy classes of subgroups $G_{z}$ in $G$. For $\phi \in M_{\mathcal{L}}$ denote by $T(\phi)$ the conjugacy class of stabilisers belonging to the unique closed orbit in $\pi^{-1}(\phi)$. Define $\left(M_{\mathcal{L}}\right)_{\tau}=\left\{\phi \in M_{\mathcal{L}}: T(\phi)=\tau\right\}$. We call $M_{\mathcal{L}}=\bigcup_{\tau \in \mathcal{T}}\left(M_{\mathcal{L}}\right)_{\tau}$ the stratification by orbit type. For each $\tau \in \mathcal{T}$ choose a stabiliser, $G_{\tau}$, representing the conjugacy class $\tau$. For all $\tau_{1}, \tau_{2} \in \mathcal{T}$ we write $G_{\tau_{1}} \leq_{c} G_{\tau_{2}}$ if $G_{\tau_{1}}$ is conjugate to a subgroup of $G_{\tau_{2}}$. We define a partial order on $\mathcal{T}$ by $\tau_{1} \geq \tau_{2}$ if $G_{\tau_{1}} \leq_{c} G_{\tau_{2}}$.

The proposition below is due to Schwarz, another proof can be found in [12].

Proposition. [28, Lemma 5.5] For each $\tau \in \mathcal{T}$ the stratum $\left(M_{\mathcal{L}}\right)_{\tau}$ is irreducible and locally closed and its closure is $\bigcup_{\nu \leq \tau}\left(M_{\mathcal{L}}\right)_{\nu}$. The stratification $M_{\mathcal{L}}=\bigcup_{\tau \in \mathcal{T}}\left(M_{\mathcal{L}}\right)_{\tau}$ is finite.

We would like to compare the stratification by orbit type to the stratification by symplectic leaves. In particular that would require that each stratum is a symplectic manifold. 
4.6. We shall need a useful lemma. For any $H \leq G$ let $Z_{(H)}=\left\{z \in Z: G_{z}\right.$ is conjugate to $\left.H\right\}$. For any $\tau \in \mathcal{T}$ such that $\left(M_{\mathcal{L}}\right)_{\tau}$ is nonempty, we choose some $H \in \tau$ and consider $Z_{(H)}$. The restriction of $\pi$ to $Z_{(H)}$ is a map whose image contains $\left(M_{\mathcal{L}}\right)_{\tau}$.

Lemma. Let $\tau \in \mathcal{T}$ and let $H \in \tau$. Suppose that $\left(M_{\mathcal{L}}\right)_{\tau} \neq \emptyset$. Then $\pi^{-1}\left(\left(M_{\mathcal{L}}\right)_{(\tau)}\right) \cap Z_{(H)}$ is a nonempty open $G$-stable subset of $Z_{(H)}$.

Proof. Let $z \in Z_{(H)}$. There exists $\sigma \in \mathcal{T}$ such that $\pi(z) \in\left(M_{\mathcal{L}}\right)_{\sigma}$. Let $X_{\sigma}=\bigcup_{\nu \leq \sigma}\left(M_{\mathcal{L}}\right)_{\nu}$ which is a closed subset of $M_{\mathcal{L}}$ by the proposition above. If the orbit $G \cdot z$ is closed in $Z$ then $\sigma=\tau$ by definition of $\left(M_{\mathcal{L}}\right)_{\sigma}$. Suppose that this orbit is not closed. Then $\sigma \neq \tau$ and the unique closed orbit in $\pi^{-1}(\pi(z))$ has dimension strictly less than $\operatorname{Dim} G \cdot z$ by 27, Corollary 13.3.1]. Let $K \in \sigma$. By comparing dimensions we see that $K \Varangle_{c} H$ and this implies that $\tau \not \leq \sigma$. Therefore $\left(M_{\mathcal{L}}\right)_{\tau} \cap X_{\sigma}=\emptyset$.

Let $\mathcal{S}=\left\{\sigma \in \mathcal{T}: \pi(z) \in\left(M_{\mathcal{L}}\right)_{\sigma}\right.$ for some $\left.z \in Z_{(H)}\right\}$ and let $\mathcal{S}^{\prime}=\mathcal{S} \backslash\{\tau\}$. By definition we have $\pi^{-1}\left(\bigcup_{\sigma \in \mathcal{S}} X_{\sigma}\right) \cap Z_{(H)}=Z_{(H)}$. By the proposition above $X_{\tau} \backslash\left(M_{\mathcal{L}}\right)_{\tau}$ is closed in $M_{\mathcal{L}}$. Therefore $\bigcup_{\sigma \in \mathcal{S}} X_{\sigma} \backslash\left(M_{\mathcal{L}}\right)_{\tau}=\left(X_{\tau} \backslash\left(M_{\mathcal{L}}\right)_{\tau}\right) \cup \bigcup_{\sigma \in \mathcal{S}^{\prime}} X_{\sigma}$ is closed in $M_{\mathcal{L}}$ and

$$
\begin{aligned}
\pi^{-1}\left(\left(M_{\mathcal{L}}\right)_{(\tau)}\right) \cap Z_{(H)} & =\left(\pi^{-1}\left(\bigcup_{\sigma \in \mathcal{S}} X_{\sigma}\right) \cap Z_{(H)}\right) \backslash\left(\pi^{-1}\left(\bigcup_{\sigma \in \mathcal{S}} X_{\sigma} \backslash\left(M_{\mathcal{L}}\right)_{\tau}\right) \cap Z_{(H)}\right) \\
& =Z_{(H)} \backslash\left(\pi^{-1}\left(\bigcup_{\sigma \in \mathcal{S}} X_{\sigma} \backslash\left(M_{\mathcal{L}}\right)_{\tau}\right) \cap Z_{(H)}\right)
\end{aligned}
$$

is open in $Z_{(H)}$. That this set is nonempty and $G$-stable follows from the definition of $Z_{(H)}$ and the fact that $\pi$ is constant on $G$-orbits.

4.7. We prove a result crucial to the proof of Theorem 1.3. The following theorem is based very closely on [29, Theorem 2.1]. This earlier result was proved over $\mathbb{R}$ and for a compact Lie group, we provide full details to verify that the result carries over to our situation.

Theorem. Let $\mathcal{L}$ be a fixed point in $\mathfrak{g}^{*}$. Then the decomposition $M_{\mathcal{L}}=\bigsqcup\left\{\left(M_{\mathcal{L}}\right)_{\tau}: \tau \in \mathcal{T}\right\}$ is a stratification of $M_{\mathcal{L}}$ into a disjoint union of symplectic manifolds. Let $\tau \in \mathcal{T}$ and choose $H \in \tau$. Then the pullback of the symplectic form, $\left(\omega_{0}\right)_{\tau}$, of $\left(M_{\mathcal{L}}\right)_{\tau}$ to $\pi^{-1}\left(\left(M_{\mathcal{L}}\right)_{\tau}\right) \cap Z_{(H)}$ equals the restriction to $\pi^{-1}\left(\left(M_{\mathcal{L}}\right)_{\tau}\right) \cap Z_{(H)}$ of the symplectic form $\omega$.

Proof. We first give the proof when $\mathcal{L}=\mathbf{0}$. Let $\zeta \in\left(M_{\mathcal{L}}\right)_{\tau}$ and $G m \subseteq Z$ be the unique closed orbit in $\pi^{-1}(\zeta)$. Let $\Upsilon=\left(G \times \mathfrak{h}^{\perp *} \times \hat{M}\right) / / H$. By Theorem 4.4 we can work in the model space $\Upsilon$, that is, there is $G$-equivariant biholomorphism, intertwining symplectic structures, between a neighbourhood of $G m$ in $M$ and a neighbourhood of $G / H \times 0 \times 0$ in $\Upsilon$.

Let $\Upsilon_{(H)}=\left\{y \in \Upsilon: G_{y}\right.$ is conjugate to $\left.H\right\}$, where $G_{y}$ denotes the stabiliser of $y$ with respect to the action of $G$ on $\Upsilon$. Let $y \in \Upsilon_{(H)}$. Then we can write $y=[g, \xi, \hat{m}]$, and for all $x \in G_{y},[x g, \xi, \hat{m}]=[g, \xi, \hat{m}]$, that is, there exists $h \in H$ such that $(x g, \xi, \hat{m})=$ $\left(g h^{-1}, \mathrm{Ad}^{*}(h) \xi, h \hat{m}\right)$. By considering the first component of $y$ we deduce that $g^{-1} G_{y} g=H$, and therefore for all $h \in H, \operatorname{Ad}^{*}(h) \xi=\xi$ and $h \hat{m}=\hat{m}$. It follows from Theorem 3.3 that $h \hat{m}=\hat{m}$ for all $h \in H$ implies $\hat{\mu}(\hat{m})=0$. Therefore

$$
\begin{aligned}
J^{-1}(0) \cap \Upsilon_{(H)} & =\left\{[g, \xi, \hat{m}]: \operatorname{Ad}^{*}(h) \xi=\xi, h \hat{m}=\hat{m} \text { for all } h \in H \text { and } \xi+\hat{\mu}(\hat{m})=0\right\} \\
& =\{[g, \xi, \hat{m}]: \xi=0 \text { and } h \hat{m}=\hat{m} \text { for all } h \in H\} \\
& =\left(G \times \hat{M}_{H}\right) / / H=G / H \times \hat{M}_{H},
\end{aligned}
$$


where $\hat{M}_{H}$ is the subspace of fixed points of $\hat{M}$, which is a symplectic subspace of $\hat{M}$.

We note that the map $\tilde{\Psi}$ from Theorem 4.4 maps points of $Z_{(H)}$ to $J^{-1}(0) \cap \Upsilon_{(H)}$. Thus the calculation of the previous paragraph and the local normal form imply that $Z_{(H)}$ is a (not necessarily connected) submanifold of $M$. By Lemma 4.6 for any nonempty open $U \subseteq\left(M_{\mathcal{L}}\right)_{\tau}$, $\pi^{-1}(U) \cap Z_{(H)}$ is a nonempty open $G$-stable subset of $Z_{(H)}$. Therefore by Theorem 4.4 we have open sets $U \subseteq\left(M_{\mathcal{L}}\right)_{\tau}$ and $W \subseteq \hat{M}_{H}$ and a commutative diagram

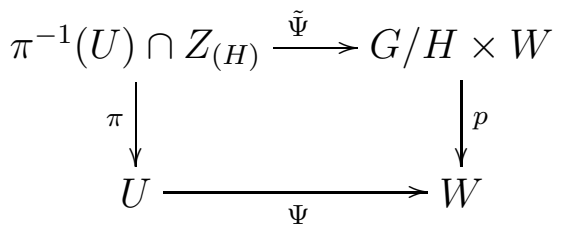

where $\Psi, \tilde{\Psi}$ are biholomorphisms, $\tilde{\Psi}$ is $G$-equivariant and intertwines the restrictions of the symplectic forms to $Z_{(H)}$ and $G / H \times \hat{M}_{H}$, and $p$ is the projection map.

Now using the map $\Psi$ we can give $\left(M_{\mathcal{L}}\right)_{\tau}$ the structure of a symplectic manifold. Note that by Proposition $4.5\left(M_{\mathcal{L}}\right)_{\tau}$ is irreducible and therefore connected. The statement about the pullback of $\left(\omega_{0}\right)_{\tau}$ follows from (2) since the pullback by $p$ of the symplectic form on $W$ equals the restriction of the symplectic form of $\Upsilon$ to $G / H \times W$.

For a general fixed point $\mathcal{L}$ we use the 'shifting trick' of Lemma 3.5 to reduce to the case above. $\mathcal{L}$ is a symplectic variety in a trivial way, so we form the product $M \times-\mathcal{L}$, which is a symplectic variety with moment map $\mu^{\prime}(m,-\mathcal{L})=\mu(m)+-\mathcal{L}$. The local normal form for $\mu^{\prime}$ will exist because it does so for $\mu$, and we can identify $\mu^{\prime-1}(\mathbf{0}) / / G$ with $\mu^{-1}(\mathcal{L}) / / G$.

4.8. We can define a bracket, $\{-,-\}_{1}$, on $\mathcal{O}\left(M_{\mathcal{L}}\right)$ in the following way. Let $f, g \in \mathcal{O}\left(M_{\mathcal{L}}\right)$ and $p \in\left(M_{\mathcal{L}}\right)_{\tau}$ for some $\tau \in \mathcal{T}$. Then $\{f, g\}_{1}(p)=\left\{\left.f\right|_{\tau},\left.g\right|_{\tau}\right\}_{\tau}(p)$ where $\left.f\right|_{\tau},\left.g\right|_{\tau}$ denote the restrictions to $\left(M_{\mathcal{L}}\right)_{\tau}$ of $f$ and $g$ respectively and $\{-,-\}_{\tau}$ is the Poisson bracket induced by the symplectic form, $\left(\omega_{0}\right)_{\tau}$. It is not clear that this is a Poisson bracket on $\mathcal{O}\left(M_{\mathcal{L}}\right)$; we remedy this below. Let $d \pi_{z}$ denote the differential of $\pi$ at $z \in Z$. For the 2 -form $\left(\omega_{0}\right)_{\tau}$ on $\left(M_{\mathcal{L}}\right)_{\tau}$ we denote by $\pi^{*}\left(\omega_{0}\right)_{\tau}$ the pullback of $\left(\omega_{0}\right)_{\tau}$ by $\pi$. The following proposition is based on [29, Proposition 3.1].

Proposition. Let $\{-,-\}_{2}$ denote the Poisson bracket on $\mathcal{O}\left(M_{\mathcal{L}}\right)$ defined in 3.4. Then $\{-,-\}_{1}=\{-,-\}_{2}$.

Proof. Let $f, g \in \mathcal{O}\left(M_{\mathcal{L}}\right)$. Let $\tau \in \mathcal{T}, H \in \tau$ and $p_{0} \in\left(M_{\mathcal{L}}\right)_{\tau}$. It suffices to show that $\{f, g\}_{1}\left(p_{0}\right)=\{f, g\}_{2}\left(p_{0}\right)$. Let $G p$ be the unique closed orbit in $\pi^{-1}\left(p_{0}\right)$ so that $p \in Z_{(H)}$. Let $\tilde{f}, \tilde{g} \in \mathcal{O}(M)^{G}$ be such that $\tilde{f}+I^{G}=f$ and $\tilde{g}+I^{G}=g$ where $I$ is the ideal of functions in $\mathcal{O}(M)$ vanishing on $Z$. Then $\{f, g\}_{2}\left(p_{0}\right)=\{\tilde{f}, \tilde{g}\}(p)$.

Let $S=\pi^{-1}\left(\left(M_{\mathcal{L}}\right)_{\tau}\right) \cap Z_{(H)}$. The proof of Theorem 4.7 shows that $S$ is a submanifold of $M$. It follows from [21, Proposition 10.5.2] that for any $z \in Z_{(H)}$ travelling along the integral curve to $\Xi_{\tilde{f}}(z)$ preserves stabiliser type, that is, this integral curve is contained in $M_{(H)}$. Proposition 3.4 implies that this integral curve is also contained in $Z$. Therefore $\Xi_{\tilde{f}}(z)$ is contained in the tangent space to $z$ in $S$.

Let $\left[\left.\tilde{f}\right|_{S}\right]$ denote the germ of $\tilde{f}$ around $p$ in $S$, and let $\left[\left.f\right|_{\tau}\right]$ denote the germ of $f$ around $p_{0}$ in $\left(M_{\mathcal{L}}\right)_{\tau}$. By Theorem 4.7 we have $\left.\omega\right|_{S}=\pi^{*}\left(\omega_{0}\right)_{(\tau)}$ and so for all $\phi \in T_{p} S$,

$$
\left(d \pi_{p}(\phi)\right)\left(\left[\left.f\right|_{\tau}\right]\right)=\phi\left(\left[\left.\tilde{f}\right|_{S}\right]\right)=\omega\left(\Xi_{\tilde{f}}(p), \phi\right)=\pi^{*}\left(\omega_{0}\right)_{(\tau)}\left(\Xi_{\tilde{f}}(p), \phi\right)=\left(\omega_{0}\right)_{(\tau)}\left(d \pi_{p}\left(\Xi_{\tilde{f}}(p)\right), d \pi_{p}(\phi)\right) .
$$


It is shown in the proof of Theorem 4.7 that the map $\pi: S \rightarrow\left(M_{\mathcal{L}}\right)_{\tau}$ is a (complex analytic) fibration of type $G / H$. Thus the differential $d \pi_{p}: T_{p} S \rightarrow T_{p_{0}}\left(\left(M_{\mathcal{L}}\right)_{\tau}\right)$ is surjective. Therefore $d \pi_{p}\left(\Xi_{\tilde{f}}(p)\right)=\Xi_{\left.f\right|_{\tau}}\left(p_{0}\right)$ (the latter Hamiltonian vector field being defined with respect to $\left.\left(\omega_{0}\right)_{(\tau)}\right)$.

By definition of the bracket $\{-,-\}_{1}$ we have $\{f, g\}_{1}\left(p_{0}\right)=\left(\omega_{0}\right)_{(\tau)}^{i}\left(\Xi_{\left.f\right|_{\tau}}\left(p_{0}\right), \Xi_{\left.g\right|_{\tau}}\left(p_{0}\right)\right)$, and then one calculates

$$
\begin{array}{r}
\{f, g\}_{1}\left(p_{0}\right)=\left(\omega_{0}\right)_{(\tau)}\left(\Xi_{\left.f\right|_{\tau}}\left(p_{0}\right), \Xi_{\left.g\right|_{\tau}}\left(p_{0}\right)\right)=\left(\omega_{0}\right)_{(\tau)}\left(d \pi_{p}\left(\Xi_{\tilde{f}}(p)\right), d \pi_{p}\left(\Xi_{\tilde{g}}(p)\right)\right) \\
\quad=\pi^{*}\left(\omega_{0}\right)_{(\tau)}\left(\Xi_{\tilde{f}}(p), \Xi_{\tilde{g}}(p)\right)=\omega\left(\Xi_{\tilde{f}}(p), \Xi_{\tilde{g}}(p)\right)=\{\tilde{f}, \tilde{g}\}(p)=\{f, g\}_{2}\left(p_{0}\right) .
\end{array}
$$

4.9. We can now describe the symplectic leaves of $M_{\mathcal{L}}$. We refer to the Poisson bracket on $\mathcal{O}\left(M_{\mathcal{L}}\right)$ from the proposition above by $\{-,-\}$.

Proposition. The orbit type strata $\left(M_{\mathcal{L}}\right)_{\tau}$ are the symplectic leaves of $M_{\mathcal{L}}$. There are finitely many symplectic leaves and they are irreducible locally closed subvarieties.

Proof. Let $\tau \in \mathcal{T}$. The set $X=\bigcup_{\nu \leq \tau}\left(M_{\mathcal{L}}\right)_{\tau}$ is closed by Proposition 4.5, Let $I$ be the ideal of functions vanishing on $X$. Let $x \in X$ so that $x \in\left(M_{\mathcal{L}}\right)_{\nu}$ for some $\nu \leq \tau$. For any $f \in \mathcal{O}\left(M_{\mathcal{L}}\right)$ and $i \in I$ we have $\{f, i\}(x)=\left\{\left.f\right|_{\nu},\left.i\right|_{\nu}\right\}_{\nu}(x)=\left\{\left.f\right|_{\nu}, 0\right\}_{\nu}(x)=0$, that is, $I$ is a Poisson ideal of $\mathcal{O}\left(M_{\mathcal{L}}\right)$. Therefore the radical of $I$ is a Poisson ideal of $\mathcal{O}\left(M_{\mathcal{L}}\right)$ by 11 , 3.3.2]. Thus $X$ is a Poisson subvariety of $M_{\mathcal{L}}$.

On the other hand, the set $X^{\prime}=X \backslash\left(M_{\mathcal{L}}\right)_{\tau}$ is closed and an identical argument to that of the previous paragraph shows that it is a Poisson subvariety of $M_{\mathcal{L}}$. Let $p \in\left(M_{\mathcal{L}}\right)_{\tau}$ and let $\mathcal{S}$ be the symplectic leaf through $p$ in $M_{\mathcal{L}}$. If $\mathcal{S} \cap X^{\prime} \neq \emptyset$ then $\mathcal{S} \subseteq X^{\prime}$ by Proposition 2.8, but this cannot happen because $p \notin X^{\prime}$. Therefore $\mathcal{S} \subseteq X \backslash X^{\prime}=\left(M_{\mathcal{L}}\right)_{\tau}$.

Since $\left(M_{\mathcal{L}}\right)_{\tau}$ is a symplectic manifold $T_{p}\left(M_{\mathcal{L}}\right)_{\tau}$ is spanned (in the notation of the proposition above) by the set $\left\{\{f,-\}_{2}(p): f \in \mathcal{O}\left(M_{\mathcal{L}}\right)\right\}$. As we have shown above, $\{f,-\}_{2}(p)=$ $\{f,-\}_{1}(p)$ for all $f \in \mathcal{O}\left(M_{\mathcal{L}}\right)$ and so $T_{p}\left(M_{\mathcal{L}}\right)_{\tau}$ is spanned by Hamiltonian vector fields for the Poisson bracket on $\mathcal{O}\left(M_{\mathcal{L}}\right)$. Our choice of $p \in\left(M_{\mathcal{L}}\right)_{\tau}$ was arbitrary and $\left(M_{\mathcal{L}}\right)_{\tau}$ is connected by Proposition 4.5 so we deduce that $\left(M_{\mathcal{L}}\right)_{\tau} \subseteq \mathcal{S}$. Therefore the $\left(M_{\mathcal{L}}\right)_{\tau}$ are the symplectic leaves of $M_{\mathcal{L}}$ and their properties are a consequence of Proposition 4.5.

\section{Deformed PREPROJECTIVE ALGEBras}

5.1. Representations of quivers. We apply the results of the previous section to representations of quivers, in particular we prove Theorem 1.3 .

Let $Q$ be a quiver with vertex set $I$, set of arrows $A$, and let $h, t: A \rightarrow I$ denote the head and tail functions respectively. If $\alpha \in \mathbb{N}^{I}$, the space of representations of $Q$ of dimension vector $\alpha$ is

$$
\operatorname{Rep}(Q, \alpha)=\bigoplus_{a \in A} \operatorname{Mat}\left(\alpha_{h(a)} \times \alpha_{t(a)}, \mathbb{C}\right)
$$

The group

$$
\mathrm{G}(\alpha)=\left(\prod_{i \in I} \mathrm{GL}\left(\alpha_{i}, \mathbb{C}\right)\right) / \mathbb{C}^{\times}
$$

acts by conjugation on $\operatorname{Rep}(Q, \alpha)$. It is clear that $\operatorname{Rep}(Q, \alpha)$ classifies representations of the path algebra $\mathbb{C} Q$ of dimension vector $\alpha$, and that the $\mathrm{G}(\alpha)$-orbits are the isomorphism classes of representations. 
5.2. Moment map. Given $\alpha \in \mathbb{N}^{I}, \operatorname{Rep}(Q, \alpha)$ is an affine space. Let $\bar{Q}$ denotes the double of $Q$ obtained from $Q$ be adjoining a reverse arrow $a^{*}: j \rightarrow i$ for each arrow $a: i \rightarrow j$ in $A$. We denote the set of arrows in $\bar{Q}$ by $\bar{A}$. Through the trace pairing,

$$
\operatorname{Rep}(\bar{Q}, \alpha)=\bigoplus_{a \in A} \operatorname{Mat}\left(\alpha_{h(a)} \times \alpha_{t(a)}, \mathbb{C}\right) \oplus \bigoplus_{a \in A} \operatorname{Mat}\left(\alpha_{h\left(a^{*}\right)} \times \alpha_{t\left(a^{*}\right)}, \mathbb{C}\right)
$$

is the cotangent bundle of $\operatorname{Rep}(Q, \alpha)$. There is a canonical symplectic form on $\operatorname{Rep}(\bar{Q}, \alpha)$ by

$$
\omega\left(\left(B_{a}, B_{a^{*}}\right),\left(C_{a}, C_{a^{*}}\right)\right)=\sum_{a \in A}-\operatorname{tr} B_{a^{*}} C_{a}+\operatorname{tr} C_{a^{*}} B_{a}
$$

for all $\left(B_{a}, B_{a^{*}}\right),\left(C_{a}, C_{a^{*}}\right) \in \operatorname{Rep}(\bar{Q}, \alpha)$.

The action of $G(\alpha)$ extends to $\operatorname{Rep}(\bar{Q}, \alpha)$ and preserves the symplectic form. By Theorem 3.3 this action is Hamiltonian and one can easily verify (see [7, Page 258] for example) that the corresponding moment map is

$$
\mu_{\alpha}: \operatorname{Rep}(\bar{Q}, \alpha) \longrightarrow \operatorname{End}(\alpha)_{0}, \quad \mu_{\alpha}\left(B_{a}, B_{a^{*}}\right)_{i}=\sum_{a \in A, h(a)=i} B_{a} B_{a^{*}}-\sum_{a \in A, t(a)=i} B_{a^{*}} B_{a}
$$

where

$$
\operatorname{End}(\alpha)_{0}=\left\{\theta \in \bigoplus_{i \in I} \operatorname{Mat}\left(\alpha_{i} \times \alpha_{i}, \mathbb{C}\right): \sum_{i \in I} \operatorname{tr}\left(\theta_{i}\right)=0\right\} \cong(\operatorname{Lie} \mathrm{G}(\alpha))^{*} .
$$

The isomorphism $\operatorname{End}(\alpha)_{0} \cong(\operatorname{Lie} \mathrm{G}(\alpha))^{*}$ is obtained via the trace pairing and is $\mathrm{G}(\alpha)$ equivariant.

Given $\lambda \in \mathbb{C}^{I}$ with $\lambda \cdot \alpha=0$, there is a $\mathrm{G}(\alpha)$-invariant element of $\operatorname{End}(\alpha)_{0}$ whose $i$ th component is $\lambda_{i} \operatorname{Id}_{\alpha_{i}}$. The corresponding Marsden-Weinstein reductions are

$$
\mathcal{N}(\lambda, \alpha)=\mu_{\alpha}^{-1}\left(\lambda_{i} \operatorname{Id}_{\alpha_{i}}\right) / / \mathrm{G}(\alpha) .
$$

5.3. Given $\lambda \in \mathbb{C}^{I}$ define the deformed preprojective algebra

$$
\Pi_{\lambda}=\frac{\mathbb{C} \bar{Q}}{\left(\sum_{a \in A}\left[a, a^{*}\right]-\sum_{i \in I} \lambda_{i} e_{i}\right)},
$$

where $\left[a, a^{*}\right]=a a^{*}-a^{*} a$. This algebra is independent of the orientation of $Q$. If $\alpha \in \mathbb{N}^{I}$ is such that $\lambda \cdot \alpha=0$ then the representations of $\Pi_{\lambda}$ of dimension $\alpha$ can be $\mathrm{G}(\alpha)$-equivariantly identified with $\mu_{\alpha}^{-1}(\lambda)$. By a result of Artin, [2, Section 12], $\mathcal{N}(\lambda, \alpha)$ classifies the isomorphism classes of semisimple representations of $\Pi_{\lambda}$ of dimension $\alpha$. It is known if $\lambda \cdot \alpha \neq 0$ then $\Pi_{\lambda}$ has no representations of dimension $\alpha$, [7] Theorem 1.2].

5.4. Stratifying $\mathcal{N}(\lambda, \alpha)$ by representation type. Recall the variety $\mathcal{N}(\lambda, \alpha)$ classifies semisimple $\Pi_{\lambda}$-modules. If $M$ is a semisimple $\Pi_{\lambda}$-module then we can decompose it into its simple components $M=M_{1}^{\oplus k_{1}} \oplus \cdots \oplus M_{r}^{\oplus k_{r}}$ where the $M_{t}$ are non-isomorphic simples. If $\beta^{(t)}$ is the dimension vector of $M_{t}$, then we say $M$ has representation type

$$
\left(k_{1}, \beta^{(1)} ; \ldots ; k_{r}, \beta^{(r)}\right),
$$

which is defined up to permutation of the entries $\left(k_{i}, \beta^{(i)}\right)$. Let $\tau=\left(k_{1}, \beta^{(1)} ; \ldots ; k_{r}, \beta^{(r)}\right)$ and let $\mathcal{R}_{\tau}$ be the subset of $\mathcal{N}(\lambda, \alpha)$ with representation type equal (up to permutation) to $\tau$. We shall refer to the stratification $\mathcal{N}(\lambda, \alpha)=\bigsqcup_{\tau ; \mathcal{R}_{\tau} \neq \emptyset} \mathcal{R}_{\tau}$ as the stratification by representation type for $\mathcal{N}(\lambda, \alpha)$. Recall the stratification by orbit type from 4.5 
Theorem. The stratification by representation type is equal to the stratification by orbit type, that is, for each $\tau, \mathcal{R}_{\tau}=\mathcal{N}(\lambda, \alpha)_{\nu}$ for some $\nu \in \mathcal{T}$.

Proof. For any quiver $Q$, the proof of [20, Theorem 2] shows that the stratification by representation type of $\operatorname{Rep}(\bar{Q}, \alpha) / / \mathrm{G}(\alpha)$ is equal to the stratification by orbit type. Since $\mathrm{G}(\alpha)$ is reductive one can identify $\mu_{\alpha}^{-1}(\lambda) / / \mathrm{G}(\alpha)$ with a closed subvariety of $\operatorname{Rep}(\bar{Q}, \alpha) / / \mathrm{G}(\alpha)$. To conclude we note that the subsets $\mathcal{R}_{\tau}, \mathcal{N}(\lambda, \alpha)$, are obtained by intersecting $\mu_{\alpha}^{-1}(\lambda) / / \mathrm{G}(\alpha)$ with the corresponding representation type and orbit type strata, respectively, of $\operatorname{Rep}(\bar{Q}, \alpha) / /$ $\mathrm{G}(\alpha)$.

5.5. Hyper-Kähler structure. Following Nakajima, 25, Section 3] and [24, Section 2], we describe the hyper-Kähler structure of $\operatorname{Rep}(\bar{Q}, \alpha)$, see 4.1. We consider the (real) manifold $\operatorname{Rep}(\bar{Q}, \alpha)$. Let $I$ denote the complex structure given by multiplication by $\sqrt{-1}$. We define a Hermitian inner product on $\operatorname{Rep}(\bar{Q}, \alpha)$ as follows. For each vertex $i$ we give each $\mathbb{C}^{\alpha_{i}}$ the standard Hermitian inner product. We get a Hermitian inner product on $\operatorname{Mat}\left(\alpha_{j} \times \alpha_{i}\right)$ by $(B, C)=\operatorname{tr}\left(B C^{\dagger}\right)$ for all $B, C \in \operatorname{Mat}\left(\alpha_{j} \times \alpha_{i}\right)$ (we use $\dagger$ to denote the Hermitian conjugate). Extending this to the whole of $\operatorname{Rep}(\bar{Q}, \alpha)$ yields a Hermitian inner product, $(-,-)$. Taking the real part of $(-,-)$ yields a real inner product which is invariant under $I$. We get further $\mathbb{R}$-linear maps by

$$
J\left(B_{a}, B_{a^{*}}\right)=\left(B_{a^{*}}^{\dagger},-B_{a}^{\dagger}\right), K=-J I
$$

and it is a straightforward check that $I^{2}=J^{2}=K^{2}=I J K=-\operatorname{Id}$. $\operatorname{Thus} \operatorname{Rep}(\bar{Q}, \alpha)$ is a hyper-Kähler manifold.

In the notation of 4.2 we have a real symplectic form $\omega_{1}$ and a complex symplectic form $\omega$. This complex symplectic form is the same as the one defined in 5.2 above. Let $K(\alpha)=$ $\prod_{k \in I} U\left(\alpha_{i}\right) / U(1)$, a maximal connected (real) subgroup of $\mathrm{G}(\alpha)$. Then $K(\alpha)$ and $\mathrm{G}(\alpha)$ act linearly on $\operatorname{Rep}(\bar{Q}, \alpha)$ and preserve $\omega_{1}$ and $\omega$ respectively. In this situation the complex moment map $\mu$ from 4.2 equals $\mu_{\alpha}$ from 5.2

5.6. We have established that $\operatorname{Rep}(\bar{Q}, \alpha)$ satisfies all of the hypotheses of Section 4 so we can apply all of the results therein. In particular we can prove Theorem 1.3 .

Proof of Theorem 1.3. By Proposition 4.9 the symplectic leaves of $\mathcal{N}(\lambda, \alpha)$ are the orbit type strata, and by Theorem 5.4 the orbit type strata equal the representation type strata.

5.7. Roots. Let $Q, A, I, h, t$ be as in 5.1. Our goal is to use Theorem 1.3 to describe the symplectic leaves of certain deformed quotient singularities. Given that the leaves of $\mathcal{N}(\lambda, \alpha)$ are described by representation types it is clear that we will need more information about the dimension vectors of simple representations. This leads us to the notion of root vectors of $Q$.

Elements of $\mathbb{Z}^{I}$ are vectors and we write $\epsilon_{i}$ for the coordinate vector at vertex $i$. We say that a vector $\alpha$ has connected support if the quiver with vertices $\left\{i \in I: \alpha_{i} \neq 0\right\}$ and arrows $\left\{a \in A: \alpha_{h(a)}, \alpha_{t(a)} \neq 0\right\}$ is connected. We partially order $\mathbb{Z}^{I}$ via $\alpha \geq \beta$ if $\alpha_{i} \geq \beta_{i}$ for all $i$, and we write $\alpha>\beta$ to mean that $\alpha \geq \beta$ and $\alpha \neq \beta$. A vector $\alpha$ is positive if $\alpha>0$, and negative if $\alpha<0$. For any subset $X \subseteq \mathbb{Z}^{I}$ we denote the positive and negative roots in $X$ by $X^{+}$and $X^{-}$respectively. We shall denote the standard inner product of $x, y \in \mathbb{C}^{I}$ by $x \cdot y$. 
The Ringel form on $\mathbb{Z}^{I}$ is defined by

$$
\langle\alpha, \beta\rangle=\sum_{i \in I} \alpha_{i} \beta_{i}-\sum_{a \in A} \alpha_{t(a)} \beta_{h(a)} .
$$

Let $(\alpha, \beta)=\langle\alpha, \beta\rangle+\langle\beta, \alpha\rangle$ be its symmetrisation. Define

$$
p(\alpha)=1+\sum_{a \in A} \alpha_{t(a)} \alpha_{h(a)}-\alpha \cdot \alpha=1-\frac{1}{2}(\alpha, \alpha) .
$$

The fundamental region, $\mathcal{F}$, is the set $0 \neq \alpha \in \mathbb{N}^{I}$ with connected support and with $\left(\alpha, \epsilon_{i}\right) \leq 0$ for every vertex $i$. If $i$ is a loopfree vertex (so $\left.p\left(\epsilon_{i}\right)=0\right)$, there is a reflection $s_{i}: \mathbb{Z}^{I} \rightarrow \mathbb{Z}^{I}$ defined by

$$
s_{i}(\alpha)=\alpha-\left(\alpha, \epsilon_{i}\right) \epsilon_{i} .
$$

The real roots, $R e$, are the elements of $\mathbb{Z}^{I}$ which can be obtained from the coordinate vector at a loopfree vertex by applying a sequence of reflections at loopfree vertices. The imaginary roots, Im, are the elements of $\mathbb{Z}^{I}$ which can be obtained from $\mathcal{F} \cup-\mathcal{F}$ by a sequence of reflections at loopfree vertices. For a quiver without loops it is easy to see that $\alpha \in R e$ implies that $p(\alpha)=0$. The set of roots is $R=\operatorname{Re} \cup \operatorname{Im}$. For $\lambda \in \mathbb{C}^{I}$ we set $R_{\lambda}=\{\alpha \in R: \lambda \cdot \alpha=0\}$.

5.8. Simple dimension vectors. For a representation type $\tau=\left(k_{1}, \beta^{(1)} ; \ldots ; k_{r}, \beta^{(r)}\right)$ (as defined in 5.4), Crawley-Boevey proved the following

Proposition. [7, Theorem 1.3] If $\mathcal{R}_{\tau} \neq \emptyset$ then $\mathcal{R}_{\tau}$ is an irreducible locally closed subset of $\mathcal{N}(\lambda, \alpha)$ of dimension $\sum_{t=1}^{r} 2 p\left(\beta^{(t)}\right)$.

In order to understand $\mathcal{N}(\lambda, \alpha)$ further we shall need to describe the dimension vectors of simple $\Pi_{\lambda}$-modules. Let $R_{\lambda}^{+}$be the set of positive roots $\beta$ with $\beta \cdot \lambda=0$.

Theorem. [7, Corollary 1.4] For $\lambda \in \mathbb{C}^{I}$ and $\alpha \in \mathbb{N}^{I}$ the following are equivalent

(1) There is a simple representation of $\Pi_{\lambda}$ of dimension vector $\alpha$;

(2) $\alpha \in R_{\lambda}^{+}$and $p(\alpha)>\sum_{t=1}^{r} p\left(\beta^{t}\right)$ for any decomposition $\alpha=\beta^{(1)}+\cdots+\beta^{(r)}$ with $r \geq 2$ and $\beta^{(t)} \in R_{\lambda}^{+}$.

In this case $\mathcal{N}(\lambda, \alpha)$ is an irreducible variety of dimension $2 p(\alpha)$ and its general element is a simple representation of $\Pi_{\lambda}$.

Henceforth we will let $\Sigma_{\lambda}$ denote the set of dimension vectors of irreducible representations of $\Pi_{\lambda}$, that is, vectors satisfying condition (2) of the above theorem.

5.9. Decomposition theorem. Let $\lambda \in \mathbb{C}^{I}$ and let $\mathbb{N} R_{\lambda}^{+}$denote the set of sums (including zero) of the elements of the set $R_{\lambda}^{+}$. Let $\alpha \in \mathbb{N} R_{\lambda}^{+}$. Define

$$
|\alpha|_{\lambda}=\max \left\{\sum_{t=1}^{r} p\left(\beta^{(t)}\right): \alpha=\sum_{t=1}^{r} \beta^{(t)} \text { with } \beta^{(t)} \in \Sigma_{\lambda} \text { for all } t\right\} .
$$

The following theorem of Crawley-Boevey reduces the study of general $\mathcal{N}(\lambda, \alpha)$ to the case where $\alpha \in \Sigma_{\lambda}$. For any variety, $X$, let $\operatorname{Sym}^{n} X$ denote the $n^{\text {th }}$ symmetric product of $X$.

Theorem. [8, Theorem 1.1] Let $\lambda \in \mathbb{C}^{I}$ and $\alpha \in \mathbb{N} R_{\lambda}^{+}$. Then 
(1) There is a unique decomposition $\alpha=\sigma^{(1)}+\cdots \sigma^{(r)}$ with $\sigma^{(t)} \in \Sigma_{\lambda}$ for all $t$, such that $|\alpha|_{\lambda}=\sum_{t=1}^{r} p\left(\sigma^{(t)}\right)$.

(2) Any other decomposition of $\alpha$ as a sum of elements of $\Sigma_{\lambda}$ is a refinement of this decomposition.

(3) Collecting terms and rewriting this decomposition as $\alpha=\sum_{t=1}^{s} m_{t} \sigma^{(t)}$ where $\sigma^{(1)}, \ldots, \sigma^{(s)}$ are distinct and $m_{1}, \ldots, m_{s}$ are positive integers, we have

$$
\mathcal{N}(\lambda, \alpha) \cong \prod_{t=1}^{s} \operatorname{Sym}^{m_{t}} \mathcal{N}\left(\lambda, \sigma^{(t)}\right)
$$

In particular $\mathcal{N}(\lambda, \alpha)$ is irreducible of dimension $2|\alpha|_{\lambda}$.

5.10. The smooth locus of $\mathcal{N}(\lambda, \alpha)$. Using the above theorem we can determine whether $\mathcal{N}(\lambda, \alpha)$ is smooth. The theorem below is known: for $\alpha \in \Sigma_{\lambda}$, Le Bruyn has proved that the smooth locus of $\mathcal{N}(\lambda, \alpha)$ is the stratum of representation type $(1, \alpha)$, [19, Theorem 3.2]. For general $\alpha$ with decomposition $\alpha=\sigma^{(1)}+\cdots \sigma^{(r)}$ as in Theorem 5.9 (1), collecting terms we have $\mathcal{N}(\lambda, \alpha) \cong \prod_{t=1}^{s} \operatorname{Sym}^{m_{t}} \mathcal{N}\left(\lambda, \sigma^{(t)}\right)$. Then one observes that for any affine algebraic variety, $X$, with $\operatorname{Dim} X \geq 2$, the smooth locus of $\operatorname{Sym}^{n} X$ is the set $\left\{\left[x_{1}, \ldots, x_{n}\right]\right.$ : the $x_{i}$ are smooth points in $X$ and $x_{i} \neq x_{j}$ for all $\left.i \neq j\right\}$. It follows that the smooth locus of $\mathcal{N}(\lambda, \alpha)$ is the stratum of representation type $\left(1, \sigma^{(1)} ; \ldots ; 1, \sigma^{(r)}\right)$. We give a proof utilising Theorem 1.3

Theorem. Let $\lambda \in \mathbb{C}^{I}$ and $\alpha \in \mathbb{N} R_{\lambda}^{+}$. The smooth locus of $\mathcal{N}(\lambda, \alpha)$ coincides with the stratum of representation type $\left(1, \sigma^{(1)} ; \ldots ; 1, \sigma^{(r)}\right)$, where $\alpha=\sigma^{(1)}+\cdots \sigma^{(r)}$ is the unique decomposition from Theorem 5.9 (1).

Proof. Let $\tau=\left(1, \sigma^{(1)} ; \ldots ; 1, \sigma^{(r)}\right)$, then $\operatorname{Dim} \mathcal{R}_{\tau}=\operatorname{Dim} \mathcal{N}(\lambda, \alpha)$ by Proposition 5.8 and Theorem [5.9 (3). Therefore $\overline{\mathcal{R}}_{\tau}=\mathcal{N}(\lambda, \alpha)$ because both varieties are irreducible. By Theorem 1.3 and [3, Proposition 3.7], $\mathcal{R}_{\tau}$ is the smooth locus of $\mathcal{N}(\lambda, \alpha)$.

In particular, we have established that the smooth locus of $\mathcal{N}(\lambda, \alpha)$ is always symplectic.

5.11. For convenience we spell out how to determine whether $\mathcal{N}(\lambda, \alpha)$ is smooth in terms of roots of $Q$. We write $\alpha=\sigma^{(1)}+\cdots \sigma^{(r)}=\sum_{i=1}^{t} m_{i} \sigma^{(i)}$, by collecting like terms.

Corollary. $\mathcal{N}(\lambda, \alpha)$ is smooth if and only if $\alpha=\sum_{i=1}^{t} m_{i} \sigma^{(i)}$ is the only possible decomposition of $\alpha$ as a sum of elements of $\Sigma_{\lambda}$ and for each $i, p\left(\sigma^{(i)}\right)>0$ implies that $m_{i}=1$.

Proof. By the theorem, $\mathcal{N}(\lambda, \alpha)$ is smooth if and only if $\left(1, \sigma^{(1)} ; \ldots ; 1, \sigma^{(r)}\right)$ is the unique representation type. The result follows from the lemma below.

Lemma. [7, Page 260] Let $\beta \in \Sigma_{\lambda}$. If $p(\beta)=0$ then there is a unique simple representation of $\Pi_{\lambda}$ with dimension vector $\beta$ (up to isomorphism); if $p(\beta)>0$ then there are infinitely many non-isomorphic simple representations with dimension vector $\beta$.

5.12. Symplectic leaves. We also show how to work out the symplectic leaves of $\mathcal{N}(\lambda, \alpha)$. These are equal to the representation type strata by Theorem 1.3 and so this task amounts to finding each of the possible decompositions of $\alpha$ as a sum of vectors in $\Sigma_{\lambda}$ and listing the representation types arising out of each decomposition.

For a decomposition

$$
\alpha=n_{1} \gamma_{1}+\ldots n_{s} \gamma_{s}+m_{1} \beta_{1}+\cdots+m_{t} \beta_{t}
$$


where $\gamma_{i}, \beta_{j} \in \Sigma_{\lambda}$ for all $i, j, p\left(\gamma_{i}\right)=0$ and $p\left(\beta_{j}\right)>0$ for all $i, j$, the corresponding representation types of $\mathcal{N}(\lambda, \alpha)$ are labeled by $t$-tuples of partitions. More precisely, let $P_{j}$ be the set of partitions of $m_{j}$ for each $j$, and for any $\sigma \in P_{j}$ denote its length by $l(\sigma)$. All the representation types coming from (3) are parametrised naturally by $P_{1} \times \cdots \times P_{t}$, thanks to Lemma 5.11, For each $\left(\sigma_{1}, \ldots, \sigma_{t}\right) \in P_{1} \times \cdots \times P_{t}$ we denote the corresponding representation type by $\tau_{\left(\sigma_{1}, \ldots, \sigma_{t}\right)}$. Then $\operatorname{Dim} \mathcal{R}_{\tau_{\left(\sigma_{1}, \ldots, \sigma_{t}\right)}}=2 \sum_{i=1}^{t} l\left(\sigma_{i}\right) p\left(\beta_{i}\right)$ by Proposition 5.8

5.13. Extended Dynkin diagrams. We give a useful lemma which applies to the quivers we consider in the proof of Theorem [1.4. Let $Q$ be an extended Dynkin diagram (see [5]), oriented to have no cycles (this is no restriction since the deformed preprojective algebras are orientation independent).

Let $\delta$ be the minimal positive imaginary root. Then $\delta$ is isotropic, that is, $(\delta, \delta)=0$ and furthermore $\left(\delta, \epsilon_{k}\right)=0$ for all $k \in I$. Any vertex for which $\delta_{k}=1$ is called an extending vertex - extending vertices always exist. We can relabel the vertex set $I$ so that 0 is an extending vertex. All real roots satisfy $(\alpha, \alpha)=1$.

Let $Q^{\prime}$ be the quiver obtained from $Q$ by adding a vertex $\infty$ and one arrow from 0 to this vertex. We'll use apostrophes to denote data associated with $Q^{\prime}$.

Lemma. Let $n \in \mathbb{N}$. Then

(1) $p^{\prime}\left(\epsilon_{\infty}+n \delta\right)=n$.

(2) If $\epsilon_{\infty}+n \delta=\beta^{(1)}+\cdots+\beta^{(r)}$, where the $\beta^{(i)}$ are positive roots, then $\sum_{t=1}^{r} p^{\prime}\left(\beta^{(t)}\right) \leq$ $p^{\prime}\left(\epsilon_{\infty}+n \delta\right)$, with equality exactly when all but one of the $\beta^{(t)}$ are equal to $\delta$.

Proof.

(1) This is a direct calculation, $p^{\prime}\left(\epsilon_{\infty}+n \delta\right)=1-\frac{1}{2}\left(\left(\epsilon_{\infty}, \epsilon_{\infty}\right)+2\left(n \delta, \epsilon_{\infty}\right)+(n \delta, n \delta)\right)=$ $1-\frac{1}{2}(2-2 n+0)=n$.

(2) This is [7, Lemma 9.2].

\section{Calogero-Moser space}

6.1. We now begin the proof of Theorem 1.4, which will occupy this and the subsequent section. We fix notation for the remainder of the paper. Let $n$ be an integer greater than 1 . Let $\left(L, \omega_{L}\right)$ be a 2 -dimensional symplectic vector space, and $\Gamma<\operatorname{Sp}(L)=\operatorname{SL}(2, \mathbb{C})$ a finite subgroup. Let $x, y \in L$ be a symplectic basis and let $R$ be the regular representation of $\Gamma$. Consider the set, $\mathbf{C}$, of all functions $\Gamma \backslash\{1\} \rightarrow \mathbb{C}$ which are constant on conjugacy classes; we identify this set with $\mathbb{C}^{d}$ where $d+1$ equals the number of conjugacy classes in $\Gamma$. For $\underline{c} \in \mathbb{C}^{d}$ and $c_{1} \in \mathbb{C}$, we shall write $\mathbf{c}=\left(c_{1}, \underline{c}\right)$.

As a $\Gamma$-module, $R^{n}$ decomposes into a sum of irreducible representations:

$$
R^{n}=\sum_{i=0}^{d} S_{i} \otimes \mathbb{C}^{n \delta_{i}}
$$

where the $S_{i}$ are the irreducible modules of $\Gamma$ and $\delta_{i}=\operatorname{Dim} S_{i}$. We shall assume that $S_{0}$ is the trivial module. It is clear from the above that one can identify the group $\operatorname{Aut}_{\Gamma}\left(R^{n}\right)$ with $\hat{G}(n \delta)=\prod_{i=0}^{d} \operatorname{GL}\left(n \delta_{i}, \mathbb{C}\right)$, where $\delta \in \mathbb{N}^{d+1}$ is the vector with $i$ th entry $\delta_{i}$. 
6.2. Calogero-Moser space. Denote by $e_{\Gamma} \in \operatorname{End}_{\Gamma}(R)$ the projector onto the trivial representation. Let $\underline{c} \in \mathbf{C}$ and $c \in \operatorname{End}_{\Gamma}(R)$ multiplication by the central element $\sum_{\Gamma \backslash\{1\}} \underline{c}(\gamma) \gamma$. Let $\mathcal{O}$ be the $\operatorname{GL}(n, \mathbb{C})$-conjugacy class formed by all $n \times n$-matrices of the form $P-\mathrm{Id}$, where $P$ is a semisimple rank one matrix such that $\operatorname{tr}(P)=\operatorname{tr}(\mathrm{Id})=n$. Define

$$
X_{\mathbf{c}}=\left\{\nabla \in \operatorname{Hom}_{\Gamma}\left(L, \operatorname{End}_{\mathbb{C}}\left(R^{n}\right)\right):[\nabla(x), \nabla(y)] \in \frac{1}{2} c_{1}|\Gamma| \mathcal{O} \otimes e_{\Gamma}+\operatorname{Id} \otimes c\right\},
$$

where $c_{1} \in \mathbb{C}$ and $\frac{1}{2} c_{1}|\Gamma| \mathcal{O} \otimes e_{\Gamma}+\operatorname{Id} \otimes c \subseteq \operatorname{End}\left(\mathbb{C}^{n}\right) \otimes \operatorname{End}_{\Gamma}(R)=\operatorname{End}_{\Gamma}\left(\mathbb{C}^{n} \otimes R\right)=\operatorname{End}_{\Gamma}\left(R^{n}\right)$.

There is an action of $\hat{\mathrm{G}}(n \delta)=\operatorname{Aut}_{\Gamma}\left(R^{n}\right)$ by basechange: let $g \in \operatorname{Aut}_{\Gamma}\left(R^{n}\right)$ and $\nabla \in X_{\mathbf{c}}$, then $(g \circ \nabla)(x)=g \nabla(x) g^{-1}$ for all $x \in L$. The action factors through $\mathbb{C}^{\times}$(where we identify $\mathbb{C}^{\times}$with $\left.\left\{\lambda \cdot \mathrm{Id}: \lambda \in \mathbb{C}^{\times}\right\}\right)$so that $\mathrm{G}(n \delta)=\hat{\mathrm{G}}(n \delta) / \mathbb{C}^{\times}$acts on $X_{\mathbf{c}}$. The variety $X_{\mathbf{c}} / / \mathrm{G}(n \delta)$ is called Calogero-Moser Space for $\Gamma_{n}$. There is a discussion of this space in [13, §11].

6.3. The vector space $\operatorname{Hom}_{\Gamma}\left(L, \operatorname{End}_{\mathbb{C}}\left(R^{n}\right)\right)=\left(L^{*} \otimes \operatorname{End}_{\mathbb{C}}\left(R^{n}\right)\right)^{\Gamma}$ is symplectic with form $\omega_{L} \otimes$ $\operatorname{tr}$, where $\operatorname{tr}$ is the symmetric bilinear form $\operatorname{tr}(\phi, \psi)=\operatorname{tr}(\phi \psi)$ for $\phi, \psi \in \operatorname{End}_{\mathbb{C}}\left(R^{n}\right)$. Clearly the action of $\mathrm{G}(n \delta)$ preserves this form. We can identify $\operatorname{End}_{\Gamma}^{0}\left(R^{n}\right):=\left\{A \in \operatorname{End}_{\mathbb{C}}\left(R^{n}\right)\right.$ : $\left.\operatorname{tr}_{R^{n}} A=0\right\}$ with $(\text { Lie } \mathrm{G}(n \delta))^{*}$ via the trace pairing. One can check that $[\nabla(x), \nabla(y)]$ : $R^{n} \rightarrow R^{n}$ is a $\Gamma$-map and that $\operatorname{tr}([\nabla(x), \nabla(y)] A)=\frac{1}{2}\left(\omega_{L} \otimes \operatorname{tr}\right)(A \cdot \nabla, \nabla)$ for all $\nabla \in$ $\operatorname{Hom}_{\Gamma}\left(L, \operatorname{End}_{\mathbb{C}}\left(R^{n}\right)\right)$ and $A \in \operatorname{End}_{\Gamma}^{0}\left(R^{n}\right)$. Therefore by Theorem 3.3 the action of $\mathrm{G}(n \delta)$ is Hamiltonian with moment map $\nabla \mapsto[\nabla(x), \nabla(y)]$ and so $X_{\mathbf{c}} / / \mathrm{G}(n \delta)$ is a Marsden-Weinstein reduction and in particular is a Poisson variety.

6.4. The shifting trick. We immediately note an equivalent form for $X_{\mathbf{c}}$. Let $\nabla \in$ $\operatorname{Hom}_{\Gamma}\left(L, \operatorname{End}_{\mathbb{C}}\left(R^{n}\right)\right)$, and let $x^{*}, y^{*}$ be a dual basis to $x, y$ so that we can write $\nabla=$ $x^{*} \otimes \phi+y^{*} \otimes \psi$ for some $\phi, \psi \in \operatorname{End}_{\mathbb{C}}\left(R^{n}\right)$. Then $[\nabla(x), \nabla(y)]=\phi \psi-\psi \phi$. In this way it is straightforward to check that $[\nabla(x), \nabla(y)]$ is the map

$$
[\nabla, \nabla]: R^{n} \stackrel{\zeta \otimes I d}{\longrightarrow} L \otimes L \otimes R^{n} \stackrel{I d \otimes \nabla}{\longrightarrow} L \otimes R^{n} \stackrel{\nabla}{\longrightarrow} R^{n}
$$

where $\zeta$ is the $\Gamma$-map

$$
\zeta: \mathbb{C} \rightarrow L \otimes L, \zeta(1)=x \otimes y-y \otimes x .
$$

By the adjunction of Hom and $\otimes$ we have a $G(n \delta)$-equivariant isomorphism between $\operatorname{Hom}_{\Gamma}\left(L, \operatorname{End}_{\mathbb{C}}\left(R^{n}\right)\right)$ and $\operatorname{Hom}_{\Gamma}\left(L \otimes R^{n}, R^{n}\right)$. Therefore

$$
X_{\mathbf{c}} / / \mathrm{G}(n \delta)=\left\{\nabla \in \operatorname{Hom}_{\Gamma}\left(L \otimes R^{n}, R^{n}\right):[\nabla, \nabla] \in \frac{1}{2} c_{1}|\Gamma| \mathcal{O} \otimes e_{\Gamma}+\mathrm{Id} \otimes c\right\} / / \mathrm{G}(n \delta) .
$$

We shall apply the shifting trick of Lemma 3.5 to the Calogero-Moser space $X_{\mathbf{c}} / / \mathrm{G}(n \delta)$. Let $m=-\frac{1}{2} n c_{1}|\Gamma|$. If $m \neq 0$ let $U_{m}$ be the $\operatorname{GL}(n, \mathbb{C})$-conjugacy class formed by all $n \times n$ matrices which are semisimple rank one and whose trace is equal to $m$. Set $U_{0}=0$. Then

$$
\begin{aligned}
X_{\mathbf{c}} / / \mathrm{G}(n \delta)=\left\{(\nabla, P) \in \operatorname{Hom}_{\Gamma}\left(L \otimes R^{n}, R^{n}\right) \times\right. & U_{m} \otimes e_{\Gamma}:[\nabla, \nabla]+P= \\
& \left.-\frac{1}{2} c_{1}|\Gamma| \operatorname{Id} \otimes e_{\Gamma}+\operatorname{Id} \otimes c\right\} / / \mathrm{G}(n \delta) .
\end{aligned}
$$


6.5. Linearized Calogero-Moser space. Ultimately we shall show that $X_{\mathbf{c}} / / \mathrm{G}(n \delta)$ is isomorphic to one of the Marsden-Weinstein reductions for quivers described in Section 5. The Calogero-Moser space is also a Marsden-Weinstein reduction but is defined over coadjoint orbit which a larger than just a single point, the latter being the case for quiver reductions. Therefore we shall give a linearized version of Calogero-Moser space. Let

$$
\begin{array}{r}
\hat{X}_{\mathbf{c}}=\left\{(\nabla, I, J) \in \operatorname{Hom}_{\Gamma}\left(L \otimes R^{n}, R^{n}\right) \oplus\left(R^{n}\right)^{\Gamma} \oplus\left(\left(R^{n}\right)^{*}\right)^{\Gamma}:[\nabla, \nabla]+(I \otimes J)\right. \\
\left.=-\frac{1}{2} c_{1}|\Gamma| \operatorname{Id} \otimes e_{\Gamma}+\operatorname{Id} \otimes c\right\}
\end{array}
$$

with the action of $\hat{\mathrm{G}}(n \delta)$ on $\hat{X}_{\mathbf{c}}$ by basechange. There is a natural symplectic form on $\operatorname{Hom}_{\Gamma}\left(L \otimes R^{n}, R^{n}\right) \oplus\left(R^{n}\right)^{\Gamma} \oplus\left(\left(R^{n}\right)^{*}\right)^{\Gamma}$ (cf. 6.2) given by $\omega\left((\nabla, I, J),\left(\nabla^{\prime}, I^{\prime}, J^{\prime}\right)\right)=\left(\omega_{L} \otimes\right.$ $\operatorname{tr})\left(\nabla, \nabla^{\prime}\right)-J\left(I^{\prime}\right)+J^{\prime}(I)=\operatorname{tr}\left[\nabla, \nabla^{\prime}\right]-J\left(I^{\prime}\right)+J^{\prime}(I)$. The action of $\mathrm{G}(n \delta)$ preserves this form so by Theorem 3.3 the action is Hamiltonian. One can easily verify that the map $\rho:(\nabla, I, J) \mapsto[\nabla, \nabla]+(I \otimes J)$ is the moment map for this action. Therefore the space $\hat{X}_{\mathbf{c}} / / \hat{\mathrm{G}}(n \delta)$ is a Marsden-Weinstein reduction.

6.6. The vector space $\left(R^{n}\right)^{\Gamma}$ is equal to $S_{0}^{n}=\mathbb{C}^{n}$. We form the symplectic vector space $\left(R^{n}\right)^{\Gamma} \oplus\left(\left(R^{n}\right)^{*}\right)^{\Gamma}$ with the obvious action of $\operatorname{GL}(n, \mathbb{C})$ preserving this form. The corresponding moment map $\nu:\left(R^{n}\right)^{\Gamma} \oplus\left(\left(R^{n}\right)^{*}\right)^{\Gamma} \rightarrow \mathfrak{g l}_{n}$ given by $(I, J) \mapsto I \otimes J$ is $\operatorname{GL}(n, \mathbb{C})$-equivariant and Poisson by Proposition 3.1. Here, as usual, we identify $\mathfrak{g l}_{n}$ with its dual via the trace pairing. Recall that $m=-\frac{1}{2} n c_{1}|\Gamma|$.

Proposition. There is an isomorphism of Poisson varieties $\hat{X}_{\mathbf{c}} / / \hat{\mathrm{G}}(n \delta) \cong X_{\mathbf{c}} / / \mathrm{G}(n \delta)$.

Proof. Suppose first that $c_{1} \neq 0$. Taking traces of the defining equation of $\hat{X}_{\mathbf{c}}$,

$$
[\nabla, \nabla]+I \otimes J=-\frac{1}{2} c_{1}|\Gamma| \operatorname{Id} \otimes e_{\Gamma}+\operatorname{Id} \otimes c,
$$

yields $\operatorname{tr}_{R^{n}}(I \otimes J)=\operatorname{tr}_{R^{n}}\left(-\frac{1}{2} c_{1}|\Gamma| \operatorname{Id} \otimes e_{\Gamma}\right)+\operatorname{tr}_{R^{n}}(\mathrm{Id} \otimes c)=m$, since $c$ is traceless on the regular representation. Therefore $I \otimes J \in \mathfrak{g l}_{n}$ is a rank one matrix with trace $m$ (which implies that it is semisimple).

Recall the notation and results from Example 3.4. In particular let $W=\mathbb{C}^{n} \oplus\left(\mathbb{C}^{n}\right)^{*}=$ $\left(R^{n}\right)^{\Gamma} \oplus\left(\left(R^{n}\right)^{*}\right)^{\Gamma}$, and let $\mu: W \rightarrow \mathbb{C}$ be the moment map for the $\mathbb{C}^{\times}$-action. Let $W_{m}$ be the fibre $\mu^{-1}(m)$. By the previous paragraph $\rho^{-1}\left(-\frac{1}{2} c_{1}|\Gamma| \operatorname{Id} \otimes e_{\Gamma}+\operatorname{Id} \otimes \mathbf{c}\right) \subseteq \operatorname{Hom}_{\Gamma}(L \otimes$ $\left.R^{n}, R^{n}\right) \times W_{m}$. The map $\rho$ is constant on $\mathbb{C}^{\times}$-orbits so induces a map $\bar{\rho}: \operatorname{Hom}_{\Gamma}\left(L \otimes R^{n}, R^{n}\right) \times$ $W_{m} / / \mathbb{C}^{\times} \rightarrow \operatorname{End}(n \delta)$ such that

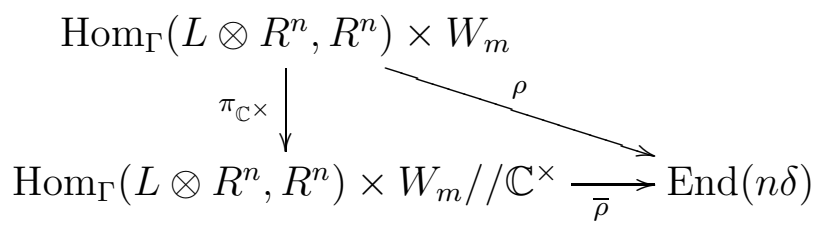

commutes. Here $\pi_{\mathbb{C}^{\times}}$is the orbit map for the $\mathbb{C}^{\times}$action. Now, $\rho$ is $\hat{\mathrm{G}}(n \delta)$-equivariant and so is $\pi_{\mathbb{C}^{\times}}$. Therefore the fact that $\pi_{\mathbb{C}^{\times}}$is surjective and $\bar{\rho} \circ \pi_{\mathbb{C}^{\times}}=\rho$ imply that $\bar{\rho}$ is $\hat{\mathrm{G}}(n \delta)$-equivariant also. 
On the other hand, recall the isomorphism $t: W_{m} / / \mathbb{C}^{\times} \rightarrow U_{m}$ from Example 3.4 this is a Poisson $\mathrm{GL}(n, \mathbb{C})$-equivariant isomorphism. We claim that the following diagram commutes

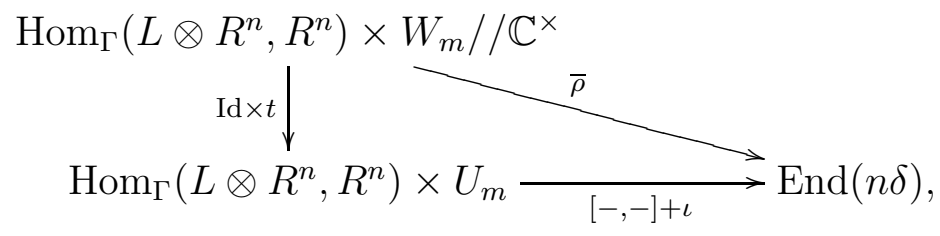

where $\iota$ is the embedding $U_{m} \hookrightarrow U_{m} \otimes e_{\Gamma} \subset \operatorname{End}(n \delta)$ and $[-,-]+\iota$ is the map taking $(\nabla, M) \in$ $\operatorname{Hom}_{\Gamma}\left(L \otimes R^{n}, R^{n}\right) \times U_{m}$ to $[\nabla, \nabla]+\iota(M)$. It follows from the surjectivity of $\pi_{\mathbb{C} \times}$ and (7) that it suffices to show that $\rho(\nabla, I, J)=([-,-]+\iota) \circ(\operatorname{Id} \times t) \circ \pi_{\mathbb{C}^{\times}}(\nabla, I, J)$ for all $(\nabla, I, J) \in$ $\operatorname{Hom}_{\Gamma}\left(L \otimes R^{n}, R^{n}\right) \times W_{m}$. However, $(\mathrm{Id} \times t) \circ \pi_{\mathbb{C}^{\times}}(\nabla, I, J)=(\nabla, I \otimes J)$ which implies that $([-,-]+\iota) \circ(\operatorname{Id} \times t) \circ \pi_{\mathbb{C}^{\times}}(\nabla, I, J)=([-,-]+\iota)(\nabla, I \otimes J)=[\nabla, \nabla]+I \otimes J=\rho(\nabla, I, J)$ as required. Therefore $\bar{\rho}$ is a moment map for the $\hat{\mathrm{G}}(n \delta)$ action by Lemma 3.5. Thus by (15) the map Id $\times t$ induces a Poisson isomorphism $\bar{\rho}^{-1}\left(-\frac{1}{2} c_{1}|\Gamma| \operatorname{Id} \otimes e_{\Gamma}+\operatorname{Id} \otimes c\right) / / \hat{\mathrm{G}}(n \delta) \cong$ $X_{\mathbf{c}} / / \mathrm{G}(n \delta)$. Furthermore the canonical isomorphism $\rho^{-1}\left(-\frac{1}{2} c_{1}|\Gamma| \operatorname{Id} \otimes e_{\Gamma}+\operatorname{Id} \otimes c\right) / / \hat{\mathrm{G}}(n \delta) \cong$ $\pi_{\mathbb{C}^{\times}}\left(\rho^{-1}\left(-\frac{1}{2} c_{1}|\Gamma| \operatorname{Id} \otimes e_{\Gamma}+\operatorname{Id} \otimes c\right)\right) / / \hat{\mathrm{G}}(n \delta)=\bar{\rho}^{-1}\left(-\frac{1}{2} c_{1}|\Gamma| \operatorname{Id} \otimes e_{\Gamma}+\operatorname{Id} \otimes c\right) / / \hat{\mathrm{G}}(n \delta)$ is Poisson by Proposition 3.4.

Now suppose that $c_{1}=0$. Then

$$
\hat{X}_{\mathbf{c}}=\left\{(\nabla, I, J) \in \operatorname{Hom}_{\Gamma}\left(L \otimes R^{n}, R^{n}\right) \oplus\left(R^{n}\right)^{\Gamma} \oplus\left(\left(R^{n}\right)^{*}\right)^{\Gamma}:[\nabla, \nabla]+(I \otimes J)=\operatorname{Id} \otimes c\right\} .
$$

Consider the closed subvariety of $\hat{X}_{\mathbf{c}}$,

$$
Z=\left\{(\nabla, I, J) \in \hat{X}_{\mathbf{c}}: I=J=0\right\} .
$$

It is clear that projecting onto the first component induces a Poisson isomorphism $Z / / \hat{\mathrm{G}}(n \delta) \cong$ $X_{\mathbf{c}} / / \mathrm{G}(n \delta)$. Therefore we have the following diagram

$$
X_{\mathbf{c}} / / \mathrm{G}(n \delta) \cong Z / / \hat{\mathrm{G}}(n \delta) \hookrightarrow \hat{X}_{\mathbf{c}} / / \hat{\mathrm{G}}(n \delta) .
$$

By Proposition $\left[7.5\right.$ there is a surjective algebra homomorphism $\phi^{*}: \mathcal{O}\left(X_{\mathbf{c}} / / \mathrm{G}(n \delta)\right) \rightarrow Z_{c}$ where the latter algebra is the centre of a symplectic reflection algebra for a wreath product and in particular has Krull dimension $2 n$, see 7.2. Therefore Dim $X_{\mathbf{c}} / / \mathrm{G}(n \delta) \geq 2 n$. On the other hand Theorem 6.10 and Lemma 6.8, which we shall prove below, imply that $\hat{X}_{\mathbf{c}} / / \hat{\mathrm{G}}(n \delta)$ is irreducible of dimension $2 n$. Therefore $Z / / \hat{\mathrm{G}}(n \delta)=\hat{X}_{\mathbf{c}} / / \hat{\mathrm{G}}(n \delta)$ and the result follows.

6.7. We now make the connection between Calogero-Moser space for $\Gamma_{n}$ and the quiver reductions from Section 5. We shall use the notation introduced in 6.1. We can associate to $\Gamma$ a graph in the following way. The McKay graph of $\Gamma$ is the graph with vertex set $I=\{0, \ldots, d\}$ and the number of edges between $i$ and $j$ is the multiplicity of $S_{i}$ in $S_{j} \otimes L$. This graph is extended of type $\tilde{A}, \tilde{D}$ or $\tilde{E}$, so it is simply laced and in particular contains no double edges, see 5 for example.

Let $Q$ be the extended Dynkin diagram corresponding to $\Gamma$ under the McKay correspondence, which is given by choosing an orientation of the McKay graph. Each vertex corresponds to an irreducible representation of $\Gamma$ and we choose $\delta \in \mathbb{N}^{I}$ so that $\delta_{i}=\operatorname{dim} S_{i}$ for all $i \in I$. This is the minimal imaginary root for $Q$ (see [5.13). 
Define a linear map

$$
\begin{aligned}
\lambda: \mathbb{C}^{d+1} \longrightarrow \mathbb{C}^{I}, \quad \mathbf{c}=\left(c_{1}, \underline{c}\right) \mapsto \lambda\left(c_{1}, \underline{c}\right)_{k} & =-\frac{1}{2} c_{1} \operatorname{tr}_{S_{k}} \sum_{\gamma \in \Gamma} \gamma+\operatorname{tr}_{S_{k}} \sum_{\gamma \in \Gamma \backslash\{1\}} \underline{c}(\gamma)(\gamma) \\
& =-\frac{1}{2} c_{1}|\Gamma| \operatorname{tr}_{S_{k}} e_{\Gamma}+\operatorname{tr}_{S_{k}} c .
\end{aligned}
$$

Now consider the quiver $Q^{\prime}$ defined by adding a vertex $\infty$ to the quiver $Q$ and adding one arrow from the vertex $\infty$ to the vertex 0 . Let $I^{\prime}=I \cup\{\infty\}$ and let $\lambda^{\prime}(\mathbf{c})=(-\lambda(\mathbf{c}) \cdot n \delta, \lambda(\mathbf{c})) \in$ $\mathbb{C}^{I^{\prime}}$. Then we have one of the Marsden-Weinstein reductions from Section 5 defined on the quiver $Q^{\prime}$ :

$$
\begin{array}{r}
\mathcal{N}\left(\lambda^{\prime}(\mathbf{c}), \epsilon_{\infty}+n \delta\right)=\left\{(x, i, j) \in \bigoplus_{a \in \bar{A}} \operatorname{Mat}\left(n \delta_{h(a)} \times n \delta_{t(a)}, \mathbb{C}\right) \oplus \operatorname{Mat}\left(n \delta_{0} \times 1, \mathbb{C}\right)\right. \\
\left.\oplus \operatorname{Mat}\left(1 \times n \delta_{0}, \mathbb{C}\right): \mu_{n \delta}(x)+i j-j i=\lambda^{\prime}(\mathbf{c})\right\} / / \mathrm{G}\left(\epsilon_{\infty}+n \delta\right) .
\end{array}
$$

Remark. As noted in [7, Section 1] $\mathcal{N}\left(\lambda^{\prime}(\mathbf{c}), \epsilon_{\infty}+n \delta\right)$ can be described as one of the quiver varieties defined by Nakajima, see [24.

6.8. We can calculate the dimension of this Marsden-Weinstein reduction.

Lemma. $\mathcal{N}\left(\lambda^{\prime}(\mathbf{c}), \epsilon_{\infty}+n \delta\right)$ is an irreducible variety of dimension $2 n$

Proof. By Theorem 5.9 (3) we know that this variety will be irreducible and have dimension $2 n$ as long as $\epsilon_{\infty}+n \delta$ is a sum of elements from $R_{\lambda^{\prime}(\mathbf{c})}^{+}$and that $\left|\epsilon_{\infty}+n \delta\right|_{\lambda^{\prime}(\mathbf{c})}=n$. Let $\beta=\epsilon_{\infty}+n \delta$. One sees that $\beta$ is in fact a root: $\left(\epsilon_{i}, \beta\right)=0$ for all $i \notin\{0, \infty\},\left(\epsilon_{0}, \beta\right)=$ $\left(\epsilon_{0}, \epsilon_{\infty}\right)=-1$ and $\left(\epsilon_{\infty}, \beta\right)=2-n \delta_{0} \leq 0$. By the definition of $|\beta|_{\lambda^{\prime}(\mathbf{c})}$ and Lemma 5.13 (2), $|\beta|_{\lambda^{\prime}(\mathbf{c})}=p^{\prime}(\beta)$ and, as noted in Lemma $5.13(1), p^{\prime}(\beta)=n$.

6.9. We state some results we shall need. Recall the map $\zeta$ from 6.4 dually, the form $\omega_{L}$ defines a $\Gamma$-map $\omega_{L}: L \otimes L \rightarrow \mathbb{C}$. By [10, Lemma 3.1] if $M$ and $N$ are $\mathbb{C} \Gamma$-modules then there is an isomorphism

$$
\operatorname{Hom}_{\Gamma}(L \otimes M, N) \rightarrow \operatorname{Hom}_{\Gamma}(M, L \otimes N) ; \phi \mapsto \phi^{b}
$$

where $\phi^{b}=(1 \otimes \phi)(\zeta \otimes 1)$. The next result is not quite the one proved in [10], but the same proof works in our situation.

Proposition. 10, Lemma 3.2] Let $Q$ be a quiver whose underlying graph is a Dynkin graph. Then one can choose $\left\{\Theta_{a}: a \in \bar{A}\right\}$ such that each $\Theta_{a}$ is a basis of $\operatorname{Hom}_{\Gamma}\left(L \otimes S_{t(a)}, S_{h(a)}\right)$ and for each $a \in A$

$$
\Theta_{a} \Theta_{a^{*}}^{b}=\frac{1}{\delta_{h(a)}} 1_{S_{h(a)}} \text { and } \Theta_{a^{*}} \Theta_{a}^{b}=-\frac{1}{\delta_{t(a)}} 1_{S_{t(a)}} .
$$

6.10. The idea of our proof of the theorem below is based on [18, Section 3].

Theorem. There is a Poisson isomorphism $\hat{X}_{\mathbf{c}} / / \hat{\mathrm{G}}(n \delta) \cong \mathcal{N}\left(\lambda^{\prime}(\mathbf{c}), \epsilon_{\infty}+n \delta\right)$.

Proof. There is an isomorphism $\mathrm{G}\left(\epsilon_{\infty}+n \delta\right) \cong \hat{\mathrm{G}}(n \delta)$ given by $\left(1, g_{i}\right) / \mathbb{C}^{\times} \mapsto\left(g_{i}\right)$ and we shall identify these two groups in what follows. We can identify $\left(R^{n}\right)^{\Gamma}$ and $\left(\left(R^{n}\right)^{*}\right)^{\Gamma}$ with 
$\operatorname{Mat}\left(n \delta_{0} \times 1, \mathbb{C}\right)$ and $\operatorname{Mat}\left(1 \times n \delta_{0}, \mathbb{C}\right)$ respectively. In this way and by using the decomposition of $R^{n},(4)$, we can describe

$$
\operatorname{Hom}_{\Gamma}\left(L \otimes R^{n}, R^{n}\right) \oplus\left(R^{n}\right)^{\Gamma} \oplus\left(\left(R^{n}\right)^{*}\right)^{\Gamma}
$$

as

$$
\operatorname{Hom}_{\Gamma}\left(\sum_{i=0}^{d} L \otimes S_{i} \otimes \mathbb{C}^{n \delta_{i}}, \sum_{i=0}^{d} S_{i} \otimes \mathbb{C}^{n \delta_{i}}\right) \oplus \operatorname{Mat}\left(n \delta_{0} \times 1, \mathbb{C}\right) \oplus \operatorname{Mat}\left(1 \times n \delta_{0}, \mathbb{C}\right)
$$

Now the definition of $Q$ from 6.7 allows us to identify this space with

$$
\begin{aligned}
\bigoplus_{a \in \bar{A}} \operatorname{Hom}_{\Gamma}\left(L \otimes S_{t(a)}, S_{h(a)}\right) \otimes \operatorname{Mat}\left(n \delta_{h(a)} \times n \delta_{t(a)}, \mathbb{C}\right) & \\
& \oplus \operatorname{Mat}\left(n \delta_{0} \times 1, \mathbb{C}\right) \oplus \operatorname{Mat}\left(1 \times n \delta_{0}, \mathbb{C}\right) .
\end{aligned}
$$

For each arrow $a \in \bar{A}$ we choose a $\Theta_{a} \in \operatorname{Hom}_{\Gamma}\left(L \otimes S_{t(a)}, S_{h(a)}\right)$ as in Proposition 6.9. There is a $\hat{\mathrm{G}}(n \delta)$-equivariant isomorphism of vector spaces, $\Psi$, from

$$
\bigoplus_{a \in \bar{A}} \operatorname{Mat}\left(n \delta_{h(a)} \times n \delta_{t(a)}, \mathbb{C}\right) \oplus \operatorname{Mat}\left(n \delta_{0} \times 1, \mathbb{C}\right) \oplus \operatorname{Mat}\left(1 \times n \delta_{0}, \mathbb{C}\right)
$$

to (91) given by

$$
\Psi\left(\left(B_{a}\right), i, j\right)=\left(\left(\Theta_{a} \otimes B_{a}\right), i, j\right)
$$

The isomorphism $\mathrm{G}\left(\epsilon_{\infty}+n \delta\right) \cong \hat{\mathrm{G}}(n \delta)$ gives an isomorphism $\operatorname{End}\left(\epsilon_{\infty}+n \delta\right)_{0} \cong \operatorname{End}(n \delta)$; $\left(M_{\infty},\left(M_{i}\right)\right) \mapsto\left(M_{i}\right)$. As noted in 6.1 we can identify the groups $\hat{\mathrm{G}}(n \delta)$ and $\operatorname{Aut}_{\Gamma}\left(R^{n}\right)$ and this yields an isomorphism of Lie algebras $\operatorname{End}(n \delta) \cong \operatorname{End}_{\Gamma} R^{n} ;\left(M_{i}\right) \mapsto\left(\operatorname{Id}_{S_{i}} \otimes M_{i}\right)$. Thus we have an isomorphism

$$
\operatorname{End}\left(\epsilon_{\infty}+n \delta\right)_{0} \cong \operatorname{End}_{\Gamma} R^{n}
$$

We claim that $\left(\left(B_{a}\right), i, j\right) \in \mu_{\epsilon_{\infty}+n \delta}^{-1}\left(\lambda^{\prime}(\mathbf{c})\right)$ if and only if $\Psi\left(\left(B_{a}\right), i, j\right) \in \hat{X}_{\mathbf{c}}$. Let $\left(\left(B_{a}\right), i, j\right) \in$ $\mu_{\epsilon_{\infty}+n \delta}^{-1}\left(\lambda^{\prime}(\mathbf{c})\right)$. In the isomorphism (10) the image of $\lambda^{\prime}(\mathbf{c})$ is $\bigoplus_{m \in I} \delta_{m}\left(-\frac{1}{2} c_{1}|\Gamma| \operatorname{Id} \otimes e_{\Gamma}+\operatorname{Id} \otimes\right.$ c) $\left.\right|_{S_{m} \otimes \mathbb{C}^{n \delta_{m}}} \in \operatorname{End}_{\Gamma} R^{n}$. Thus to prove that $\Psi\left(\left(B_{a}\right), i, j\right) \in \hat{X}_{\mathbf{c}}$ we need only verify that for each $k \in I$,

$$
\left.\left[\left(\Theta_{a} \otimes B_{a}\right),\left(\Theta_{a} \otimes B_{a}\right)\right]\right|_{S_{k} \otimes \mathbb{C}^{n \delta_{k}}}=\frac{1}{\delta_{k}} 1_{S_{k}} \otimes\left(\sum_{a \in Q, h(a)=k} B_{a} B_{a^{*}}-\sum_{a \in Q, t(a)=k} B_{a^{*}} B_{a}\right) .
$$


Let $r_{k} \otimes v_{k} \in S_{k} \otimes \mathbb{C}^{n \delta_{k}}$, then

$$
\begin{aligned}
& {\left[\left(\Theta_{a} \otimes B_{a}\right),\left(\Theta_{a} \otimes B_{a}\right)\right]\left(r_{k} \otimes v_{k}\right)=\left(\Theta \otimes B_{a}\right) \circ\left(\mathrm{Id} \otimes\left(\Theta_{a} \otimes B_{a}\right)\right)\left((x \otimes y-y \otimes x) \otimes r_{k} \otimes v_{k}\right)} \\
& =\left(\Theta_{a} \otimes B_{a}\right)\left(\sum_{\substack{a \in \bar{A}, t(a)=k}} x \otimes \Theta_{a}\left(y \otimes r_{k}\right) \otimes B_{a}\left(v_{k}\right)-\sum_{\substack{a \in \bar{A}, t(a)=k}} y \otimes \Theta_{a}\left(x \otimes r_{k}\right) \otimes B_{a}\left(v_{k}\right)\right) \\
& =\sum_{\substack{a \in \bar{A}, t(a)=k}} \sum_{\substack{b \in \bar{A}, t(b)=h(a)}} \Theta_{b}\left(x \otimes \Theta_{a}\left(y \otimes r_{k}\right)\right) \otimes B_{b}\left(B_{a}\left(v_{k}\right)\right) \\
& -\sum_{\substack{a \in \bar{A}, t(a)=k \\
t(b)=h(a)}} \Theta_{b}\left(y \otimes \Theta_{a}\left(x \otimes r_{k}\right)\right) \otimes B_{b}\left(B_{a}\left(v_{k}\right)\right) \\
& =\sum_{\substack{a \in \bar{A}, t(a)=k}} \sum_{\substack{b \in \bar{A}, t(b)=h(a)}}\left[\Theta_{b}\left(x \otimes \Theta_{a}\left(y \otimes r_{k}\right)-y \otimes \Theta_{a}\left(x \otimes r_{k}\right)\right)\right] \otimes B_{b}\left(B_{a}\left(v_{k}\right)\right) .
\end{aligned}
$$

Because $\left[\left(\Theta_{a} \otimes B_{a}\right),\left(\Theta_{a} \otimes B_{a}\right)\right]$ is a $\Gamma$-map we must have that the $b$ 's appearing in (11) have $h(b)=k$ and since $Q$ is a simply laced quiver it follows that $b=a^{*}$. Therefore (111) equals:

$$
\begin{aligned}
& \sum_{\substack{a \in \bar{A}, t(a)=k}}\left[\Theta_{a^{*}}\left(x \otimes \Theta_{a}\left(y \otimes r_{k}\right)-y \otimes \Theta_{a}\left(x \otimes r_{k}\right)\right)\right] \otimes B_{a^{*}}\left(B_{a}\left(v_{k}\right)\right) \\
& =\sum_{\substack{a \in \bar{A}, t(a)=k}}\left[\Theta_{a^{*}}\left(1 \otimes \Theta_{a}\right)\left(x \otimes y \otimes r_{k}-y \otimes x \otimes r_{k}\right)\right] \otimes B_{a^{*}}\left(B_{a}\left(v_{k}\right)\right) \\
& =\sum_{\substack{a \in \bar{A}, t(a)=k}} \Theta_{a^{*}} \Theta_{a}^{b}\left(r_{k}\right) \otimes B_{a^{*}}\left(B_{a}\left(v_{k}\right)\right) .
\end{aligned}
$$

Now by Proposition 6.9 this equals

$$
\left(\frac{1}{\delta_{k}} 1_{S_{k}} \otimes\left(\sum_{a \in A, h(a)=k} B_{a} B_{a^{*}}-\sum_{a \in A, t(a)=k} B_{a^{*}} B_{a}\right)\right)\left(r_{k} \otimes v_{k}\right),
$$

as required. On the other hand, the above calculation shows that if $\Psi\left(\left(B_{a}\right), i, j\right) \in \hat{X}_{\mathbf{c}}$ then for all $k \in I$ we have $\mu_{\epsilon_{\infty}+n \delta}\left(\left(B_{a}\right), i, j\right)_{k}=\lambda(\mathbf{c})_{k}$. It remains to show that $-j i=-\lambda(\mathbf{c}) \cdot n \delta$. We note that $\mu_{\epsilon_{\infty}+n \delta}\left(\left(B_{a}\right), i, j\right)=\mu_{n \delta}\left(B_{a}\right)+i j-j i$. Now since $\mu_{n \delta}\left(B_{a}\right)$ is traceless on $\bigoplus_{k \in I} \mathbb{C}^{n \delta_{i}}$ we get $j i=\operatorname{tr} i j=\operatorname{tr}\left(\mu_{n \delta}\left(B_{a}\right)+i j\right)=\operatorname{tr} \lambda(\mathbf{c})=\lambda(\mathbf{c}) \cdot n \delta$.

Therefore $\Psi$ induces an isomorphism of $\mu_{\epsilon_{\infty}+n \delta}^{-1}\left(\lambda^{\prime}(\mathbf{c})\right)$ to $\hat{X}_{\mathbf{c}}$, and since $\Psi$ is equivariant for the group actions we get an induced isomorphism

$$
\tilde{\Psi}: \mathcal{N}\left(\lambda^{\prime}(\mathbf{c}), \epsilon_{\infty}+n \delta\right) \rightarrow \hat{X}_{\mathbf{c}} / / \hat{\mathrm{G}}(n \delta) .
$$

We show that $\tilde{\Psi}$ is Poisson. For all $\left(\left(B_{a}\right), i, j\right),\left(\left(B_{a}^{\prime}\right), i^{\prime}, j^{\prime}\right) \in \operatorname{Rep}\left(\overline{Q^{\prime}}, \epsilon_{\infty}+n \delta\right)$,

$$
\left.\omega\left(\left(B_{a}\right), i, j\right),\left(\left(B_{a}^{\prime}\right), i^{\prime}, j^{\prime}\right)\right)=\sum_{k \in I}\left(\sum_{a \in A ; t(a)=k}-\operatorname{tr}\left(B_{a^{*}} B_{a}^{\prime}\right)+\sum_{a \in A ; h(a)=k} \operatorname{tr}\left(B_{a^{*}}^{\prime} B_{a}\right)\right)+\operatorname{tr}\left(j^{\prime} i-j i^{\prime}\right)
$$


which by an easy modification of the calculations above is equal to

$$
\sum_{k \in I} t r_{S_{k} \otimes \mathbb{C}^{n \delta_{k}}}\left[\left(\Theta \otimes B_{a}\right),\left(\Theta \otimes B_{a}^{\prime}\right)\right]+\operatorname{tr}\left(i \otimes j^{\prime}-i^{\prime} \otimes j\right) .
$$

By the description of the symplectic form on $\operatorname{Hom}_{\Gamma}\left(L \otimes R^{n}, R^{n}\right) \oplus\left(R^{n}\right)^{\Gamma} \oplus\left(\left(R^{n}\right)^{*}\right)^{\Gamma}$ from 6.5 it follows that $\Psi$ intertwines symplectic forms. Thus by Proposition $3.4 \tilde{\Psi}$ is a Poisson isomorphism.

\section{Symplectic Reflection Algebras}

7.1. Definition. Let $\left(L, \omega_{L}\right)$ be as in 6.1. The wreath product $\Gamma_{n}=S_{n} \ltimes \Gamma^{n}$ acts on $V:=L^{\oplus n}=\mathbb{C}^{n} \otimes L$, preserving the symplectic form $\omega=\omega_{L}^{n}$.

We say that $\gamma \in \Gamma_{n}$ is a symplectic reflection if $\operatorname{Dim}(1-\gamma)(V)=2$. Let $S$ denote the set of all symplectic reflections. Let c $: S \rightarrow \mathbb{C} ; \gamma \mapsto c_{\gamma}$ be constant on conjugacy classes. Given $\gamma \in S$ define the form $\omega_{\gamma}$ on $V$ to have radical $\operatorname{ker}(1-\gamma)$ and to be the restriction of $\omega$ on $(1-\gamma)(V)$.

Let $T V$ be the tensor algebra of $V$. The symplectic reflection algebra $H_{\mathbf{c}}$ is the $\mathbb{C}$-algebra defined as the quotient of the skew group ring $T V * \Gamma_{n}$ by the relations

$$
v \otimes w-w \otimes v=\sum_{\gamma \in S} c_{\gamma} \omega_{\gamma}(v, w) \gamma
$$

for all $v, w \in V$.

Usually, symplectic reflection algebras depend on a further parameter $t \in \mathbb{C}$. The definition above is the $t=0$ case.

7.2. The geometry of the centres. Let $Z_{\mathbf{c}}$ denote the centre of $H_{\mathbf{c}}$. By the PBW theorem [13. Theorem 1.3], GKdim $H_{\mathbf{c}}=2 n . H_{\mathbf{c}}$ is a finitely generated module over $Z_{\mathbf{c}}$, [13, Theorem 3.1], and it follows that $G K \operatorname{dim} Z_{\mathbf{c}}=2 n$. By the Artin-Tate Lemma, [23, Lemma 13.9.10], $Z_{\mathbf{c}}$ is a finitely generated algebra over $\mathbb{C}$; also, it follows from [13, Theorem 3.3] that $Z_{\mathbf{c}}$ is a domain. Thus, $\operatorname{Max} Z_{\mathbf{c}}$ is an irreducible variety of dimension $2 n$, and since $\operatorname{Max} Z_{\mathbf{0}}=V / \Gamma_{n}$ the varieties $\operatorname{Max} Z_{\mathbf{c}}$ form a flat family of deformations of the symplectic quotient singularity $V / \Gamma_{n}$, [13, Theorem 3.1]. The case $n=1$ is the Kleinian singularity case and that is dealt with in detail in [5].

By [13. Section 15] the algebras $Z_{\mathbf{c}}$ have a Poisson bracket which is a deformation of the bracket on $\mathcal{O}\left(V / \Gamma_{n}\right)$. Our interest is in determining for which values of $\mathbf{c}$ the varieties $\operatorname{Max} Z_{\mathbf{c}}$ are smooth, and in the singular cases calculating the symplectic leaves, which have a leading role in the representation theory of $H_{\mathbf{c}}$.

7.3. Symplectic reflections. We list the symplectic reflections of $\Gamma_{n}$ for $n>1$. Given $\gamma \in \Gamma$, we write $\gamma_{i} \in \Gamma_{n}$ for the element $\gamma$ regarded as an element of the $i$-th factor $\Gamma$. Let $s_{i j} \in S_{n}$ denote the transposition swapping $i$ and $j$. Then the group $\Gamma_{n}$ is generated by the symplectic reflections $s_{i j}$ and $\gamma_{i}$. The conjugacy classes of symplectic reflections in $\Gamma_{n}<\operatorname{Sp}(V)$ are known to be of two types:

(1) The set $\left\{s_{i j} \cdot \gamma_{i} \cdot \gamma_{j}^{-1}: i, j \in[1, n], i \neq j, \gamma \in \Gamma\right\}$ forms a single $\Gamma_{n}$ conjugacy class;

(2) The set $\left\{\gamma_{i}: i \in[1, n], \gamma \in \mathcal{C}\right\}$ forms one $\Gamma_{n}$ conjugacy class for any given conjugacy class, $\mathcal{C}$, in $\Gamma \backslash\{1\}$. 
In particular, we can identify the set of class functions $S \rightarrow \mathbb{C}$ with $\mathbb{C}^{d+1}$ where $d+1$ is the number of conjugacy classes in $\Gamma$. Therefore the flat family of (centres of) symplectic reflection algebras is parametrised by elements $\mathbf{c} \in \mathbb{C}^{d+1}$. We will assume that $\mathbf{c}=\left(c_{1}, \underline{c}\right)$ where $c_{1} \in \mathbb{C}$ corresponds to the conjugacy class (1) above and $\underline{c} \in \mathbb{C}^{d}$ corresponds to class functions $\Gamma \backslash\{1\} \rightarrow \mathbb{C}$ cf. 6.7

7.4. Let $\operatorname{Max} Z_{\mathbf{c}}$ be the variety associated to $H_{\mathbf{c}}$ as in 7.2 , The theorem we now prove is based very closely on [13, Theorem 11.16]. We make use of Lemma 11.15 in [13] as in the earlier proof, but a check shows that $c_{1}$ should be replaced by $\frac{1}{2} c_{1}$. This accounts for the discrepancy between our definition of Calogero-Moser space, $X_{\mathbf{c}} / / \mathrm{G}(n \delta)$, given in 6.2 and that given in [13, Definition 11.5].

Theorem. $\operatorname{Max} Z_{\mathbf{c}}$ and $X_{\mathbf{c}} / / \mathrm{G}(n \delta)$ are isomorphic as Poisson varieties (up to nonzero scalar multiple).

Proof. We first show that $X_{c} / / \mathrm{G}(n \delta)$ is isomorphic to Max $Z_{\mathbf{c}}$. Our argument follows that of [13, Theorem 11.16] which proves that these varieties are isomorphic for generic values of c. We show that the generic hypothesis can be removed.

Let $\operatorname{Rep}_{\Gamma_{n}}\left(H_{\mathbf{c}}\right)$ be the variety of $H_{\mathbf{c}}$-modules which are isomorphic to the regular representation of $\Gamma_{n}$. Let $e \in \mathbb{C} \Gamma_{n}$ be the symmetrising idempotent. Given $M \in \operatorname{Rep}_{\Gamma_{n}}\left(H_{\mathbf{c}}\right), e M$, the space of $\Gamma_{n}$ fixed points, is a one dimensional subspace of $M$ fixed under the action of $Z_{\mathbf{c}}$. This induces a morphism of algebraic varieties

$$
\pi: \operatorname{Rep}_{\Gamma_{n}}\left(H_{\mathbf{c}}\right) \longrightarrow \operatorname{Max} Z_{\mathbf{c}} ; M \mapsto \operatorname{ann}_{Z_{\mathbf{c}}}(e M)
$$

The group $\operatorname{Aut}_{\Gamma_{n}}\left(\mathbb{C} \Gamma_{n}\right)$ acts naturally on $\operatorname{Rep}_{\Gamma_{n}}\left(H_{\mathbf{c}}\right)$. By 13. Theorem 3.7] there is a unique irreducible component $\operatorname{Rep}_{\Gamma_{n}}^{o}\left(H_{\mathbf{c}}\right)$ of the variety $\operatorname{Rep}_{\Gamma_{n}}\left(H_{\mathbf{c}}\right)$ whose image is dense in $\operatorname{Max} Z_{\mathbf{c}}$ and whose generic point is a simple $H_{\mathbf{c}}$-module. Moreover, there is an isomorphism

$$
\pi^{*}: Z_{\mathbf{c}} \longrightarrow \mathcal{O}\left(\operatorname{Rep}_{\Gamma_{n}}^{o}\left(H_{\mathbf{c}}\right)\right)^{\operatorname{Aut}_{\Gamma_{n}}\left(C \Gamma_{n}\right)} .
$$

On the other hand, thanks to [13, pp.311], there is a morphism

$$
\psi: \operatorname{Rep}_{\Gamma_{n}}\left(H_{\mathbf{c}}\right) \longrightarrow X_{\mathbf{c}} .
$$

This morphism is obtained as follows. Let $S_{n-1} \ltimes \Gamma^{n-1}=\Gamma_{n-1}<\Gamma_{n}$, where $S_{n-1}$ fixes the label $1 \in\{1, \ldots, n\}$. Then, for $v \in L$, the element $v_{1}=(v, 0, \ldots, 0) \in L^{\oplus n} \subset H_{\mathbf{c}}$ commutes with $\Gamma_{n-1}$. Let $M \in \operatorname{Rep}_{\Gamma_{n}}\left(H_{\mathbf{c}}\right)$, and consider the $\Gamma_{n-1}$ fixed points $M^{\Gamma_{n-1}} \subseteq M$. We have a $\Gamma$-equivariant map

$$
\nabla_{M}: L \rightarrow \operatorname{End}_{\mathbb{C}}\left(M^{\Gamma_{n-1}}\right) ;\left.v \mapsto v_{1}\right|_{M^{\Gamma_{n-1}}} .
$$

Now $\psi$ sends $M$ to $\nabla_{M}$. By [13, Lemma 11.15] this is a well-defined map from $\operatorname{Rep}_{\Gamma_{n}}\left(H_{\mathbf{c}}\right)$ to $X_{\mathbf{c}}$.

The morphism $\psi$ intertwines the $\operatorname{Aut}_{\Gamma_{n}}\left(\mathbb{C} \Gamma_{n}\right)$ and $\mathrm{G}(n \delta)$ actions, and hence induces a map

$$
\phi^{*}: \mathcal{O}\left(X_{\mathbf{c}} / / \mathrm{G}(n \delta)\right) \longrightarrow \mathcal{O}\left(\operatorname{Rep}_{\Gamma_{n}}^{o}\left(H_{\mathbf{c}}\right)\right)^{\operatorname{Aut}_{\Gamma_{n}}\left(\mathbb{C} \Gamma_{n}\right)} \cong Z_{\mathbf{c}}
$$

By Proposition 7.5 below $\phi^{*}$ is surjective. However, the proof given in 13 that $\phi^{*}$ is injective requires the generic hypothesis on $\mathbf{c}$. We circumvent this in the following way: by combining Lemma 6.8. Proposition 6.6] and Theorem 6.10 we have that $X_{\mathbf{c}} / / \mathrm{G}(n \delta)$ is an irreducible variety of dimension $2 n$. The variety $\operatorname{Max} Z_{\mathbf{c}}$ is also irreducible of dimension $2 n$ and therefore the surjective morphism $\phi^{*}$ must be injective also. Therefore $X_{\mathbf{c}} / / \mathrm{G}(n \delta) \cong \operatorname{Max} Z_{\mathbf{c}}$. 
To see that this isomorphism is Poisson (up to nonzero scalar multiple) we simply note that the penultimate two paragraphs of [13, Lemma 11.18] apply word-for-word: the map $\phi^{*}$ is a filtration preserving isomorphism, and the Poisson bracket on $X_{\mathbf{c}} / / \mathrm{G}(n \delta)$ has filtration degree $\leq 2$ (in the sense of [13, Page 267]). Therefore, by [13, Lemma 2.26], $\phi^{*}$ is Poisson up to nonzero scalar multiple.

7.5. There is a result used in the proof of Theorem 7.4 which we require in the proof of Proposition 6.6. The proof given in [13] can be applied verbatim to our situation.

Proposition. [13, Theorem 11.16] The algebra homomorphism $\phi^{*}$ from (12) is a surjective map.

One consequence of Theorem 7.4 is that the isomorphism $\operatorname{Max} Z_{\mathbf{c}} \cong X_{c} / / \mathrm{G}(n \delta)$ maps symplectic leaves to symplectic leaves, [22, Lemma 3.4].

\section{EXAMPLES}

8.1. The symmetric group. We calculate some examples of Theorem [1.4 working out for which values of $\mathbf{c}$ the variety $\operatorname{Max} Z_{\mathbf{c}}$ is smooth. By [3, Proposition 3.7 and Theorem 7.8], Max $Z_{\mathbf{c}}$ is smooth if and only if it is symplectic and so there will only be nontrivial leaves when $\operatorname{Max} Z_{\mathbf{c}}$ is singular. In these cases we work out the number and dimension of the symplectic leaves. We refer the reader to the notation introduced in 7.1 .

The simplest case to consider is when the group $\Gamma$ is trivial, that is, where the there is only the symmetric group $S_{n}$ acting by its permutation representation on $\left(\mathbb{C}^{2}\right)^{n}$. Then the parameter $\mathbf{c}$ is simply a complex number. For generic values of $\mathbf{c}$ the variety $\operatorname{Max} Z_{\mathbf{c}}$ is smooth by [13, Corollary 1.14]. It is clear from the relations that $H_{t \mathbf{c}} \cong H_{\mathbf{c}}$ for all $t \neq 0$, thus $\operatorname{Max} Z_{t \mathbf{c}} \cong \operatorname{Max} Z_{\mathbf{c}}$ and we deduce that Max $Z_{\mathbf{c}}$ is smooth for all nonzero $\mathbf{c}$. It is possible to show that in this case $\operatorname{Max} Z_{\mathbf{c}}$ is isomorphic to $\mathbb{A}^{2} \times \operatorname{Max} \mathfrak{Z}_{\mathbf{c}}$, where $\mathfrak{Z}_{\mathbf{c}}$ is the centre of the rational Cherednik algebra (at $t=0)$ of type $\mathbf{A}_{n-1}$, see [13, Section 4].

8.2. The hyperoctrahedral group. The next simplest case is $\Gamma_{n}=S_{n} \ltimes\left(\mathbb{Z}_{2} \times \cdots \times \mathbb{Z}_{2}\right)$ where we identify $\mathbb{Z}_{2}$ with $<\gamma=-\operatorname{Id}_{\mathbb{C}^{2}}>$. We consider the family of varieties $\operatorname{Max} Z_{\mathbf{c}}$ where the deformation parameter is given by two variables $\mathbf{c}=\left(c_{1}, c_{\gamma}\right)$, corresponding to the conjugacy classes of symplectic reflections in $\Gamma_{n}(7.3)$. Here Max $Z_{\mathbf{c}}$ is isomorphic to Max $\mathfrak{Z}_{\mathbf{c}}$ where $\mathfrak{Z}_{\mathrm{c}}$ is the centre of the rational Cherednik algebra of type $\mathbf{B}_{n}$.

Theorem 1.4 gives us $\operatorname{Max} Z_{\mathbf{c}} \cong \mathcal{N}\left(\lambda^{\prime}(\mathbf{c}), \epsilon_{\infty}+n \delta\right)$ and this isomorphism identifies symplectic leaves. The latter is the Marsden-Weinstein reduction associated to the quiver

$$
\mathrm{Q}^{\prime}: \infty \longrightarrow 0 \rightleftarrows 1
$$

with dimension vector $\epsilon_{\infty}+n \delta=(1, n, n)$ and at parameter $\lambda^{\prime}(\mathbf{c})=\left(n c_{1},-c_{1}+c_{\gamma},-c_{\gamma}\right)$ (see 6.7). We note that it is well known that the variety $\operatorname{Max} Z_{\mathbf{c}}$ is singular when $\mathbf{c}$ equals zero and its symplectic leaves have been calculated, [3, Proposition 7.4]. We omit the proof of the following theorem, details of which can be found in the author's $\mathrm{PhD}$ thesis.

Theorem. Let $\operatorname{Max} Z_{\mathbf{c}}$ be as above and assume that $\mathbf{c} \neq(0,0)$. Then

(1) $\operatorname{Max} Z_{\mathbf{c}}$ is singular if and only if $c_{1}=0$ or $c_{\gamma}= \pm m c_{1}$ for some integer, $m$, such that $0 \leq m \leq n-1$. 
(2) If $c_{1}=0$ then the symplectic leaves of $\operatorname{Max} Z_{\mathbf{c}}$ are parametrised by $P_{n}$, the set of partitions of $n$. For each $\sigma \in P_{n}$, the corresponding leaf, $\mathcal{S}_{\sigma}$ has dimension $2 l(\sigma)$, where $l(\sigma)$ is the length of $\sigma$.

(3) If $c_{\gamma}= \pm m c_{1}$ for some $0 \leq m \leq n-1$ then the leaves are parametrised by the set $S=\left\{k \in \mathbb{Z}_{\geq 0}: k m+k^{2} \leq n\right\}$. For $k \in S$ the corresponding leaf, $\mathcal{S}_{k}$ has dimension $2\left(n-k m-k^{2}\right)$.

Remark. The behaviour of $\operatorname{Max} Z_{\mathbf{c}}$ when $c_{1}=0$ is always analogous to that occurring in the above theorem. Denote by $H_{\underline{c}}$ the symplectic reflection algebra associated to the pair $\left(L, \omega_{L}\right)$ acted on by the group $\Gamma$ (see 6.1) , with centre $Z_{\underline{c}}$. When $c_{1}=0$ it is easy to calculate that $H_{\mathbf{c}}$, the algebra defined on the wreath product, $\Gamma_{n}$, is isomorphic to $\left(\bigotimes_{n} H_{\underline{c}}\right) * S_{n}$. Therefore one can see that $\operatorname{Max} Z_{\mathbf{c}} \cong \operatorname{Sym}^{n} \operatorname{Max} Z_{\underline{c}}$. This is identical to the behaviour of symplectic reflection algebras for wreath products in the case that $t \neq 0$, see [14].

Acknowledgements The author wishes to thank Iain Gordon for suggesting this topic and contributing many helpful ideas and comments. Many thanks also go to Ken Brown for his suggestions and advice. This paper will form part of the author's $\mathrm{PhD}$ thesis and he gratefully acknowledges the support of the University of Glasgow. Part of the work for this paper was during a workshop at the University of Edinburgh in June 2005, which was supported by Leverhulme Research Interchange Grant F/00158/X.

\section{REFERENCES}

[1] M. Adler, P. van Moerbeke, and P. Vanhaecke. Algebraic integrability, Painlevé geometry and Lie algebras, volume 47 of Ergebnisse der Mathematik und ihrer Grenzgebiete. 3. Folge. A Series of Modern Surveys in Mathematics. Springer-Verlag, Berlin, 2004.

[2] M. Artin. On Azumaya algebras and finite dimensional representations of rings. J. Algebra, 11:532-563, 1969.

[3] K.A. Brown and I. Gordon. Poisson orders, symplectic reflection algebras and representation theory. $J$. reine angew. Math., 559:193-216, 2003.

[4] W. Bruns and U. Vetter. Determinantal rings, volume 1327 of Lecture Notes in Mathematics. SpringerVerlag, Berlin, 1988.

[5] H. Cassens and P. Slodowy. On Kleinian singularities and quivers. In Singularities (Oberwolfach, 1996), volume 162 of Progr. Math., pages 263-288. Birkhäuser, 1998.

[6] N. Chriss and V. Ginzburg. Representation Theory and Complex Geometry. Birkhäuser, 1997.

[7] W. Crawley-Boevey. Geometry of the moment map for representations of quivers. Compositio Math., 126(3):257-293, 2001.

[8] W. Crawley-Boevey. Decomposition of Marsden-Weinstein reductions for representations of quivers. Compositio Math., 130(2):225-239, 2002.

[9] W. Crawley-Boevey, P. Etingof, and V. Ginzburg. Noncommutative geometry and quiver algebras. AG/0502301.

[10] W. Crawley-Boevey and M.P. Holland. Noncommutative deformations of kleinian singularities. Duke Math. J., 92(3):605-635, 1998.

[11] J. Dixmier. Enveloping Algebras, volume 11 of Grad. Stud. Math. Amer. Math. Soc. Providence, RI, 1996.

[12] M. Domokos and A. N. Zubkov. Semisimple representations of quivers in characteristic p. Algebr. Represent. Theory, 5(3):305-317, 2002.

[13] P. Etingof and V. Ginzburg. Symplectic reflection algebras, Calogero-Moser space, and deformed HarishChandra homomorphism. Invent. Math, 147(2):243-348, 2002.

[14] W. L. Gan and V. Ginzburg. Deformed preprojective algebras and symplectic reflection algebras for wreath products. J. Algebra, 283(1):350-363, 2005. 
[15] V. Guillemin and S. Sternberg. Symplectic Techniques in Physics. Cambridge University Press, Cambridge, 1984.

[16] N. Hitchin. Hyper-Kähler manifolds. (206):Exp. No. 748, 3, 137-166, 1992. Séminaire Bourbaki, Vol. $1991 / 92$.

[17] A. A. Kirillov. Local Lie algebras. Uspehi Mat. Nauk, 31(4(190)):57-76, 1976.

[18] A. Kuznetsov. Quiver varieties and Hilbert schemes. AG/0111092.

[19] L. Le Bruyn. Noncommutative smoothness and coadjoint orbits. J. Algebra, 258(1):60-70, 2002.

[20] L. Le Bruyn and C. Procesi. Semisimple representations of quivers. Trans. Amer. Math. Soc., 317(2):585$598,1990$.

[21] J.E. Marsden and T.S. Ratiu. Introduction to Mechanics and Symmetry, volume 17 of Texts in Applied Mathematics. Springer-Verlag, New York, 1994.

[22] M. Martino. The associated variety of a Poisson prime ideal. J. London Math. Soc. (2), 72(1):110-120, 2005.

[23] J.C. McConnell and Robson J.C. Noncommutative Noetherian Rings. Wiley-Interscience, New York, 1987.

[24] H. Nakajima. Instantons on ALE spaces, quiver varieties, and Kac-Moody algebras. Duke Math. J., $76(2): 365-416,1994$.

[25] H. Nakajima. Lectures on Hilbert Schemes of Points on Surfaces, volume 18 of University Lecture Series. American Mathematical Society, Providence, RI, 1999.

[26] H. Nakajima. Quiver varieties and finite-dimensional representations of quantum affine algebras. $J$. Amer. Math. Soc., 14(1):145-238 (electronic), 2001.

[27] W. F. Santos and A. Rittatore. Actions and invariants of algebraic groups, volume 269 of Pure and Applied Mathematics (Boca Raton). Chapman \& Hall/CRC, Boca Raton, FL, 2005.

[28] G. W. Schwarz. Lifting smooth homotopies of orbit spaces. Inst. Hautes Études Sci. Publ. Math., (51):37$135,1980$.

[29] R. Sjamaar and E. Lerman. Stratified symplectic spaces and reduction. Ann. of Math. (2), 134(2):375$422,1991$.

[30] M. Van den Bergh. Double poisson algebras. QA/0410528.

[31] A. Weinstein. The local structure of Poisson manifolds. J. Differential Geom., 18(3):523-557, 1983.

Department of Mathematics, University of Glasgow, Glasgow, G12 8QW, U.K.

E-mail address: mma@maths.gla.ac.uk 